\title{
Loss of Mecp2 Causes Atypical Synaptic and Molecular Plasticity of Parvalbumin-Expressing Interneurons Reflecting Rett Syndrome-Like Sensorimotor Defects
}

\author{
DDoemi Morello, ${ }^{1}$ Riccardo Schina, ${ }^{1}$ Federica Pilotto, ${ }^{1}$ Mary Phillips, ${ }^{2}$ CRiccardo Melani, ${ }^{3}$ Ornella \\ Plicato, ${ }^{1}$ Tommaso Pizzorusso, ${ }^{3,4}$ (D) Lucas Pozzo-Miller, ${ }^{2}$ and PMaurizio Giustetto $^{1,5}$
}

\section{DOI:http://dx.doi.org/10.1523/ENEURO.0086-18.2018}

${ }^{1}$ Department of Neuroscience, University of Turin, Corso M. D'Azeglio 52, Turin, 10126, Italy, ${ }^{2}$ Department of Neurobiology, University of Alabama at Birmingham, Birmingham, AL 35294, USA, ${ }^{3}$ Department of Neuroscience, Psychology, Drug Research and Child Health NEUROFARBA, University of Florence, Area San Salvi Pad. 26, Florence, 50135, Italy, ${ }^{4}$ Institute of Neuroscience, National Research Council (CNR), via Moruzzi 1, Pisa, 56124, Italy, and ${ }^{5}$ National Institute of Neuroscience-Italy, Corso M. D’Azeglio 52, Turin, 10126, Italy

\begin{abstract}
Rett syndrome (RTT) is caused in most cases by loss-of-function mutations in the X-linked gene encoding methyl CpG-binding protein 2 (MECP2). Understanding the pathological processes impacting sensory-motor control represents a major challenge for clinical management of individuals affected by RTT, but the underlying molecular and neuronal modifications remain unclear. We find that symptomatic male Mecp2 knockout (KO) mice show atypically elevated parvalbumin (PV) expression in both somatosensory (S1) and motor (M1) cortices together with excessive excitatory inputs converging onto PV-expressing interneurons (INs). In accordance, high-speed voltage-sensitive dye imaging shows reduced amplitude and spatial spread of synaptically induced neuronal depolarizations in S1 of Mecp2 KO mice. Moreover, motor learning-dependent changes of PV expression and structural synaptic plasticity typically occurring on $\mathrm{PV}^{+}$INs in $\mathrm{M} 1$ are impaired in symptomatic Mecp2 KO mice. Finally, we find similar abnormalities of PV networks plasticity in symptomatic female Mecp2 heterozygous mice. These results indicate that in Mecp2 mutant mice the configuration of $\mathrm{PV}^{+}$INs network is shifted toward an atypical plasticity state in relevant cortical areas compatible with the sensory-motor dysfunctions characteristics of RTT.
\end{abstract}

Key words: Cerebral cortex; neuroanatomy; parvalbumin-expressing interneurons; Rett syndrome; structural synaptic plasticity; X-linked intellectual disability

\section{Significance Statement}

Understanding the pathologic processes impacting somatosensory processing and motor control represents a major challenge for clinical management of individuals affected by Rett syndrome. We found that Mecp2 is important, starting from a young age, for the correct molecular and synaptic organization of parvalbumin-positive interneurons in cortical areas responsible for sensory-motor skills. Intriguingly, even partial Mecp2 loss produces an atypical upregulation of parvalbumin expression in these cells that correlates with the severity of motor behavioral impairments and is associated with defective cortical activity in $\mathrm{KO}$ mice. Because in behaviorally impaired Mecp2 KO mice excessive activity-dependent excitatory connectivity is established with these interneurons, our study suggests that partial inhibition of parvalbuminpositive cells would be beneficial for motor impairments in Rett syndrome. 


\section{Introduction}

Classic Rett syndrome (RTT) is a childhood neurologic disorder affecting $\sim 1$ in 10,000 live female births that results from loss-of-function mutations in the X-linked gene MECP2 (methyl-CpG-binding protein 2), encoding a multifunctional protein that regulates gene expression and chromatin architecture by interacting with methylated nucleotides (Amir et al., 1999; Lyst and Bird, 2015). The clinical symptoms currently used as diagnostic criteria for $\mathrm{RTT}$ include an early neurologic regression, occurring after a period of typical development, that severely affects motor, cognitive, and communication skills (Smeets et al., 2012). Among the most severe clinical signs associated with RTT are the partial or complete loss of acquired purposeful hand skills and spoken language, the development of gait abnormalities (diagnosed as dyspraxia or apraxia), stereotypic hand movements, and the progressive deterioration of motor abilities (Temudo et al., 2008; Neul et al., 2010; Downs et al., 2016) until walking is completely prevented in many RTT individuals. Although sensory-motor deficits are considered among the most debilitating symptoms of RTT individuals, experimental studies providing insights into the underlying pathophysiological mechanisms are needed.

To investigate the neuropathology produced by the lack of Mecp2, several models have been generated including the Mecp2 $2^{\mathrm{tm} 1.1 \mathrm{Jae}}-$ null model, used in this study, which present early onset of the neurologic symptoms characteristic of RTT (Chen et al., 2001). On the other hand, due to random X-chromosome inactivation, female Mecp2 heterozygous (Het) mice recapitulate the cellular mosaicism of Mecp2 expression found in RTT individuals and closely phenocopy the human condition, including the typical regression of acquired behavioral abilities (Katz et al., 2012; Samaco et al., 2013). Although Mecp2 is ubiquitously expressed, brain-specific deletion of Mecp2 in mice entirely recapitulates RTT-like phenotypes, suggesting that its function is most critical in brain cells (Chen et al., 2001; Guy et al., 2001). Moreover, recent studies revealed that loss of Mecp2 in specific neuronal subtypes leads to distinct RTT-like symptoms (Chao et al., 2010; Zhang et al., 2014), with GABAergic interneurons (INs) emerging as major players in RTT pathophysiology, espe-

Received December 21, 2017; accepted August 2, 2018; First published September 11, 2018.

The authors declare no competing financial interests.

Author contributions: M.G., L.P.-M., T.P., and N.M. designed research; N.M., R.S., F.P., M.P., R.M., and O.P. performed research; N.M., R.S., F.P., M.P., R.M., and O.P. analyzed data; M.G., L.P.-M., T.P., and N.M. wrote the paper.

This work was supported by grants from AIRETT ONLUS (to M.G. and T.P.), Rettsyndrome.org n. 2814 (to M.G.), National Institutes of Health (grant numbers NS-065027, HD-074418 to L.P.-M.), and the Summer Program in Neurobiology (SPIN; to R.S.).

Correspondence should be addressed to Maurizio Giustetto, Dip. di Neuroscienze, C.so M. D'Azeglio 52, 10126, Torino, Italy. E-mail: maurizio.giustetto@unito.it.

DOI:http://dx.doi.org/10.1523/ENEURO.0086-18.2018

Copyright (C) 2018 Morello et al.

This is an open-access article distributed under the terms of the Creative Commons Attribution 4.0 International license, which permits unrestricted use, distribution and reproduction in any medium provided that the original work is properly attributed. cially parvalbumin-positive $\left(\mathrm{PV}^{+}\right)$INs (Durand et al., 2012; Tomassy et al., 2014; Krishnan et al., 2015; Banerjee et al., 2016; Krishnan et al., 2017). Indeed, mice with conditional deletion of $M e c p 2$ in $\mathrm{PV}^{+}$INs develop a wide range of functional and behavioral $\mathrm{RTT}$-like symptoms $(\mathrm{He}$ et al., 2014; Ito-Ishida et al., 2015), including atypical sensory-motor learning. $\mathrm{PV}^{+} \mathrm{IN}$ activity regulates highorder processing in the cortex by generating precisely timed inhibition/disinhibition of output target neurons (Zhang and Sun, 2011; Pi et al., 2013). Importantly, the plasticity of $\mathrm{PV}^{+} \mathrm{IN}$ responses controls the activity of specific neocortical circuits and contributes to sensory and motor information processing during behavioral learning (Cardin et al., 2009; Isomura et al., 2009; Sachidhanandam et al., 2016). For instance, $\mathrm{PV}^{+} \mathrm{INs}$ in the motor cortex exhibit cellular and molecular plasticity when a mouse learns a novel sensory-motor task, such as the rotarod test. This plasticity consists of a shift toward reduced levels of PV, identified as "low-PV-network configuration," that is caused by changes in the ratio of excitatory and inhibitory boutons converging onto $\mathrm{PV}^{+} \mathrm{INS}$. Recently, subpopulations of functionally distinct $\mathrm{PV}$ cells have been classified based on levels of PV expression (Donato et al., 2013, 2015; Caroni, 2015). Importantly, an altered PV-network configuration interferes with experience-dependent plasticity mechanism in the brain and is associated with defective motor learning (Donato et al., 2013).

Because Mecp2 mutant mice show severe motor deficits, we hypothesized that lack of MeCP2 may cause an atypical excitatory/inhibitory connectivity balance onto $\mathrm{PV}^{+}$INs which could alter the configuration of PV networks in the sensory-motor cortices affecting behavioral responses. To test this idea, we first analyzed both the expression of PV and the organization of excitatory and inhibitory synaptic inputs converging onto INs in somatosensory and motor cortices of symptomatic male Mecp2 $\mathrm{KO}$ mice. Next, we investigated these properties in presymptomatic Mecp2 KO mice before the appearance of overt pathologic symptoms (De Filippis et al., 2010). We then characterized both amplitude and spatial spread of synaptically induced neuronal depolarizations in acute cortical slices to test the impact of the lack of Mecp2 on activity before and after symptom appearance. Moreover, we evaluated learning-induced changes of PV expression and synaptic connectivity in the M1 cortex of Mecp2 KO mice. Finally, we studied the developmental trajectory of PV-network configuration and learning-dependent plasticity in female Mecp2 heterozygous mice. Our results disclose cellular and molecular alterations affecting $\mathrm{PV}^{+}$ INs in the sensory-motor cortices that could be responsible for the deficits in motor performance and learning shown by Mecp2 mutant mice.

\section{Materials and Methods}

\section{Animals}

All animal procedures were performed in accordance with the European Community Council Directive 2010/ 63/UE for care and use of experimental animals and were approved by the Italian Ministry of Health (Authorization Nr. 175/2015-PR) and by the Bioethical Committee of the 
University of Turin. At the University of Alabama at Birmingham (UAB), animals were handled and housed according to the Committee on Laboratory Animal Resources of the US National Institutes of Health, and following protocols reviewed annually by the Institutional Animal Use and Care Committee (IACUC-09021). All efforts were made to minimize animal suffering and to reduce the number of animals used. Experimental male Mecp2 knockout (Mecp2 $\mathrm{KO}$ ), female Mecp2 heterozygous mice (Mecp2 Het), and wild-type (WT) mice used for this study were obtained by crossing female Mecp2 Het mice (MMRRC Cat\# 000011UCD, RRID:MMRRC_000011-UCD; Mecp2 ${ }^{\text {tm1.1.1Jae }}$ with male WT C57BL/6J mice for one generation, followed by breeding among offspring and maintaining on a mixed background (Chen et al., 2001). At UAB, Mecp2 $2^{\text {tm1.1 Jae }}$ mice were maintained on a pure C57BL/6J background. Age-matched WT littermates were used in all experimental conditions to avoid possible consequences of genetic background unrelated to the Mecp2 mutation. All analyses were conducted by an investigator blinded to both genotype and training of mice.

\section{Voltage-sensitive dye (VSD) imaging and extracellular field recordings}

For VSD imaging and extracellular recordings, presymptomatic [postnatal day 24 (P24)-P26] and symptomatic (P45-P60) Mecp2 KO mice and WT littermates were anesthetized with ketamine (100 mg/kg i.p.) and transcardially perfused with ice-cold "cutting" artificial cerebrospinal fluid (aCSF) containing (in mM): $87 \mathrm{NaCl}, 2.5 \mathrm{KCl}, 1.25$ $\mathrm{NaH}_{2} \mathrm{PO}_{4}, 0.5 \mathrm{CaCl}_{2}, 7 \mathrm{MgCl}_{2}, 25 \mathrm{NaHCO}_{3}, 25$ glucose, 75 sucrose saturated with $95 \% \mathrm{O}_{2} / 5 \%$ CO. $300-\mu \mathrm{m}-$ thick slices from the $S 1$ cortex (bregma $-0.70-1.70$ $\mathrm{mm}$ ) were prepared using a vibratome (Leica VT1200S). Slices were incubated at $32^{\circ} \mathrm{C}$ for $30 \mathrm{~min}$ and subsequently allowed to recover for at least $1 \mathrm{~h}$ at room temperature (RT) in recording aCSF containing (in $\mathrm{mM}$ ): $125 \mathrm{NaCl}, 25 \mathrm{NaHCO}_{3}, 25$ glucose, $2.5 \mathrm{KCl}, 1.25$ $\mathrm{NaH}_{2} \mathrm{PO}_{4}, 2 \mathrm{CaCl}_{2}$, and $1 \mathrm{MgCl}_{2}$ and were saturated with $95 \% \mathrm{O}_{2} / 5 \% \mathrm{CO}_{2}$. After recovery, slices were incubated for $45 \mathrm{~min}$ at RT with the voltage-sensitive fluorescent dye $\mathrm{RH}-414$ at $30 \mu \mathrm{M}$ diluted in recording aCSF. Excess dye was washed for at least $20 \mathrm{~min}$ in an immersion chamber perfused $(2 \mathrm{~mL} / \mathrm{min})$ with recording aCSF at RT. RH-414 (absorption $532 \mathrm{~nm}$, emission $716 \mathrm{~nm}$ ) was excited with $530 \pm 50-n m$ light from a phosphor-pumped LED engine (Heliophor, 89North), and its filtered emission (580 beamsplitter, 594nm long-pass) imaged with a $10 \times(0.5 \mathrm{NA})$ Plan-Neofluar objective (Carl Zeiss) in an inverted microscope (Olympus IX-71), and acquired with a scientific CMOS camera running at 2500 frames per second $(128 \times$ 128 pixels; NeuroCMOS-SM128 Red Shirt Imaging). Extracellular field excitatory postsynaptic potentials (fEPSPs) were evoked by stimulation of layer $V$ in somatosensory cortex (S1) with a bipolar electrode using constant current pulses $(0.1 \mathrm{~ms}, 0-120 \mu \mathrm{A})$ from an isolated stimulator (ISO-Flex, AMPI), and recording in layer II/III using an Axoclamp-2A amplifier (Molecular Devices). Inputoutput relationships of VSD signals were obtained using stimulation intensities that evoked fEPSPs of $25 \%, 50 \%$,
$80 \%$, and $100 \%$ of maximum responses without a population spike. Data were analyzed using custom written Matlab (Mathworks) scripts. The amplitude of VSD signals is expressed as $\Delta F / F$, and spatio-temporal spread calculated as percentage of cortical layers I-V showing responses above a threshold of $1.5 \times 10^{-3} \Delta F / F$. Percentage of cortical area was used to account for reduced cortical thickness observed in Mecp2 KO mice (Fukuda et al., 2005). VSD signals used to compare spatial spread over time were evoked at stimulation intensities eliciting $80 \%$ of the maximum fEPSP amplitude. The number of slices and animals for each group was as follows: 12 slices from 4 WT and 24 slices from 6 Mecp2 KO mice at P45-P60; 17 slices from 3 WT and 3 Mecp2 KO mice at P24-P26. The areas under the curve of spatial spread over time were compared with Student's $t$ test, and all other comparisons were performed by two-way ANOVA with Bonferroni's post hoc analysis.

\section{Motor behavioral test: accelerating rotarod}

Two-month-old male Mecp2 KO mice, 8-mo-old female Mecp2 Het mice, and age-matched WT littermates were tested for motor learning, using an accelerating rotarod apparatus (Ugo Basile). The mice were randomly assigned to the accelerating rotarod (RR test) or to the activity control test (AC test). Mice were tested for two consecutive days, four trials each day, with a 5-min rest interval between trials. The test started when mice were placed and stable on the rod, routed to running forward while the rod was rotating. Each RR trial lasted for a maximum of 5 $\mathrm{min}$, during which the rod accelerated linearly from 4 to 40 rpm, while in AC test a constant speed of $4 \mathrm{rpm}$ was maintained. The time that it took for each mouse to fall from the rod (latency to fall) was recorded for each trial. If the mouse held on to the rod and rotated $360^{\circ}$, this time was noted, and the time of the second rotation was reported as the time of falling off the rod. For immunofluorescence experiment mice were sacrificed $2 \mathrm{~h}$ after the last rotarod session. Data were analyzed with a two-way ANOVA and Bonferroni's post hoc analysis (genotype $x$ trial).

\section{Immunofluorescence}

Animals were anesthetized with an intraperitoneal injection of a mixture of Telazol (Zoletil: $80 \mathrm{mg} / \mathrm{kg}$, Alcyon) and Xylazine (Nerfasin 2\%: $10 \mathrm{mg} / \mathrm{kg}$, Alcyon) and transcardially perfused with PBS followed by ice-cold paraformaldehyde [ $4 \%$ in $0.1 \mathrm{M}$ phosphate buffer (PB), pH 7.4]. After perfusion, brains were dissected and kept in the same fixative solution overnight at $4^{\circ} \mathrm{C}$. After several washes in PB $0.1 \mathrm{M}$, the brains were cryoprotected by immersion in $10 \%, 20 \%$, and $30 \%$ sucrose solutions and cut in $30-\mu \mathrm{m}$ coronal sections with a cryostat. Free-floating cryosections were stored at $-20^{\circ} \mathrm{C}$ in a solution containing $30 \%$ ethylene glycol and $25 \%$ glycerol in $0.1 \mathrm{M} \mathrm{PB}(\mathrm{pH} 7.2)$ until used. For free-floating immunostaining, following a blocking step in a PBS solution containing $0.05 \%$ Triton X-100 and $10 \%$ normal donkey serum (NDS), sections were incubated overnight at RT with the following primary antibodies diluted in PBS with $0.05 \%$ Triton X-100 and 3\% NDS: rabbit anti-calretinin (Synaptic Systems Cat\# 214 102 Lot\# RRID:AB_2228331;1:2000), guinea pig anti- 
calretinin (Synaptic Systems Cat\# 214104 Lot\# RRID: AB_10635160; 1:500), mouse anti-parvalbumin (Swant Cat\# 235 Lot\# RRID:AB_10000343; 1:10000), guinea pig antiVGLUT1 (Millipore Cat\# AB5905 Lot\# RRID:AB_2301751; 1:5000), and rabbit anti-VGAT (Synaptic Systems Cat\# 131003 Lot\# RRID:AB_887869; 1:1000). Double immunofluorescence was performed with simultaneous addition of the primary antibodies. Sections were washed in PBS $(3 \times 10 \mathrm{~min})$ and incubated for $1 \mathrm{~h}$ at RT with the following fluorescent secondary antibodies at 1/1000 dilution: anti-guinea pig Alexa Fluor 488 (Jackson ImmunoResearch Labs Cat\# 706-545-148 Lot\# RRID:AB_2340472), anti-rabbit Cy3 (Jackson ImmunoResearch Labs Cat\# 711-165-152 Lot\# RRID:AB_2307443), anti-mouse Cy3 (Jackson ImmunoResearch Labs Cat\# 715165-151 Lot\# RRID:AB_2315777), anti-rabbit Cy5 (Jackson ImmunoResearch Labs Cat\# 711-175-152 Lot\# RRID: AB_2340607), and anti-guinea pig Cy3 (Jackson ImmunoResearch Labs Cat\# 706-165-148 Lot\# RRID:AB_2340460). Sections were counterstained with the nuclear dye DAPI (nuclear diamidino-2-phenylindole staining, Sigma). After several PBS rinses, the sections were mounted on glass slides and imaged in a wide-field epifluorescence microscope (Eclipse 800, Nikon) equipped with a CCD camera (Axiocam HRc, Carl Zeiss) or in a confocal microscope (Zeiss LSM-5 Pascal) by using sequential acquisition of separate wavelength channels to avoid fluorescence crosstalk.

\section{Quantification of excitatory and inhibitory presynaptic boutons}

To image VGLUT1- and VGAT-positive presynaptic boutons onto PV- and CR-expressing cells, we used the confocal microscope with a $100 \times$ oil-immersion objective $(1.40 \mathrm{NA})$ and the pinhole set at 1.0 Airy unit. Each confocal image was composed of 3-4 optical sections spaced $0.5 \mu \mathrm{m}$. A minimum of 10 images per animal acquired from layer II/III of the S1 or M1 cortices were used. Images were processed for background subtraction and smoothing filtering with Imaris software (RRID: SCR_007370; release 4.2, Bitplane). Presynaptic puncta that contacted immunolabeled cell bodies and those in close juxtaposition to immunolabeled dendrites were identified as presynaptic varicosities by visual examination in three orthogonal planes using Imaris. Puncta immunolabeled for VGLUT1 or VGAT were considered to be in close apposition to either PV- or CR-positive somata or dendrites when there were no black pixels between the pre- and postsynaptic structures, as described in Pizzo et al. (2016). The number of immunolabeled presynaptic puncta was counted manually using Imaris, and they were included in the analysis only if present in at least two consecutive optical sections in the 3D $z$-axis confocal image stack. The density of immunopuncta (puncta/ $\mu \mathrm{m}$ ) was calculated by measuring the length of dendritic segments or the perimeter of cell bodies contacted by the axonal terminals using the measuring tool in ImageJ software (RRID:SCR_003070, National Institutes of Health).

\section{PV immunoreactivity: cell density and signal intensity}

To examine PV immunoreactivity, we acquired images from at least 3 coronal brain sections that included $\mathrm{S} 1$ or M1 cortices according to a mouse brain atlas (Franklin and Paxinos, 1997) from Mecp2 KO and WT mice using an epifluorescence microscope (Eclipse 800, Nikon) with a $10 \times$ objective. Digital boxes spanning from the pia surface to the white matter (corpus callosum) were superimposed at matched locations on each coronal section of the cerebral cortex. Layer II/III area was identified based on DAPI staining as a cytoarchitectonic reference and measured using ImageJ. A square frame of $80 \times 80 \mu \mathrm{m}$ was placed in the region of the corpus callosum to measure background values, and the background average was subtracted from each section. The total density of PV-immunolabeled cells and their mean fluorescence intensity, measured as average pixel intensity of gray values $(0-255)$ in each cell body, was quantified automatically using the ImageJ Plugin Nucleus Counter. First, we analyzed cumulative distributions of $\mathrm{PV}^{+}$cell intensity for each group of animals. We next classified $\mathrm{PV}^{+}$cells into four subclasses, based on PV fluorescence intensity for each condition and cortical area, as follows: we calculated lower $(I)$ and higher $(h)$ PV intensity values for the WT group (i.e., the means of the three lower and three higher values for each WT mice) and the intermediate (i) value between $/$ and $h$ values. These values were next used to determine the range of intensities in which the four subclasses of $\mathrm{PV}^{+}$INs were subdivided as follows: low-PV 0 (gray value) - I; medium-low PV I - $i$; medium-high PV $i$ $h$; high-PV $h-255$ (gray value). Data were expressed as fraction (\%) of $\mathrm{PV}^{+}$INs or cells $/ \mathrm{mm}^{2}$ when total density was statistically different between genotypes. Cumulative frequency distributions data were compared using MannWhitney $U$ test, and the fraction of $\mathrm{PV}^{+}$neurons were compared using Student's $t$ test or two-way ANOVA.

\section{Statistical analysis}

Averages of multiple measurements are presented as mean \pm SEM. For each experiment, $n$ values are indicated in the figure legends, where $n$ stands for the number of animals unless otherwise indicated. Data were statistically analyzed using unpaired two-tailed Student's $t$ test, twoway ANOVA with Bonferroni's post hoc analysis, MannWhitney $U$ and Pearson correlation tests as indicated in figure legends and in Materials and Methods subsections. All the statistical analyses were performed using Prism software (Graphpad, RRID:SCR_002798), and probability values lower than 0.05 were considered statistically significant. Statistical test results are included in Table 1. Power analysis of the statistical tests was performed using G*Power 3.1.9.2 (RRID:SCR_013726) or SPSS Statistic 22 (RRID:SCR_002865). The power of the statistical tests is reported in Table 2.

\section{Results}

\section{Age-dependent increase of the fraction of INs expressing higher levels of PV in S1 cortex of Mecp2 KO mice \\ $\mathrm{PV}^{+}$basket cells provide local feed-forward and feed-} back inhibition onto principal excitatory neurons as well as contributing to the generation of gamma oscillations that are crucial for sensory processing in neocortical circuits (Cardin et al., 2009; Sohal et al., 2009). We therefore 
Table 1. $P$ values of the indicated statistical comparisons.

\begin{tabular}{lll}
\hline Figure & Measurement & Type of test \\
Fig. $1 B$ & PV cell density & Unpaired $t$ test \\
Fig. $1 C$ & PV fluorescence intensity & Unpaired $t$ test \\
Fig. $1 D$ & Relative frequency of PV INs & Mann-Whitney $U$ test \\
Fig. $1 E$ & Fraction of PV INs & Unpaired $t$ test
\end{tabular}

Comparison

WT vs. Mecp2 KO

WT vs. Mecp2 KO

WT vs. Mecp2 KO

WT vs. Mecp2 KO (high PV)

WT vs. Mecp2 KO (medium-high PV)

WT vs. Mecp2 KO (medium-low PV)

WT vs. Mecp2 KO (low PV)

Fig. $1 G$

PV cell density

PV fluorescence intensity

Fig. $1 /$

Fig. $1 \mathrm{~J}$ Relative frequency of PV INs Fraction of PV INs

Fig. $2 E$

Fig. $2 F$

Fig. $2 G$

Fig. $2 \mathrm{H}$

Fig. $2 M$

Fig. $2 N$

Fig. 20

Fig. $2 P$

Fig. $3 E$

Fig. $3 F$

Fig. $3 G$

Fig. $3 H$

Fig. $3 M$

Fig. $3 N$

Fig. 30

Fig. $3 P$

Fig. $4 C$

Fig. $4 D$

Fig. $4 E$

Fig. $4 G$

Fig. $4 \mathrm{H}$

Fig. 4 I

Fig. $5 B$

Fig. $5 C$

Fig. $5 D$

Fig. $5 E$

Fig. $5 G$

Fig. $5 H$

Fig. 5 I

Fig. $5 \mathrm{~J}$

Fig. $6 A$

Rotarod task

Fig. $6 C$

Relative frequency of PV INs

Fig. $6 D$

Fraction of PV INs

Fraction of PV INs

Fig. $6 D$

Correlation between \% High PV and RR performance

Fig. 7B VGLUT1 density on PV dendrites

VGLUT1 density on PV soma

VGAT density on PV dendrites

Relative frequency of PV INs
Unpaired $t$ test

Unpaired $t$ test

Mann-Whitney $U$ test

Unpaired $t$ test

Unpaired $t$ test

Unpaired $t$ test

Unpaired $t$ test

Unpaired $t$ test

Unpaired $t$ test

Unpaired $t$ test

Unpaired $t$ test

Unpaired $t$ test

Unpaired $t$ test

Unpaired $t$ test

Unpaired $t$ test

Unpaired $t$ test

Unpaired $t$ test

Unpaired $t$ test

Unpaired $t$ test

Unpaired $t$ test

Two-way ANOVA

Two-way ANOVA

Unpaired $t$ test

Two-way ANOVA

Two-way ANOVA

Unpaired $t$ test

Unpaired $t$ test

Unpaired $t$ test

Mann-Whitney $U$ test

Unpaired $t$ test

Unpaired $t$ test

Unpaired $t$ test

Mann-Whitney $U$ test

Unpaired $t$ test

Two-way ANOVA

Mann-Whitney $U$ test

Two-way ANOVA

Two-way ANOVA

Pearson's $r$

Two-way ANOVA

Two-way ANOVA

Two-way ANOVA

Mann-Whitney $U$ test
WT vs. Mecp2 KO

WT vs. Mecp2 KO

WT vs. Mecp2 KO

WT vs. Mecp2 KO (high PV)

WT vs. Mecp2 KO (medium-high PV)

WT vs. Mecp2 KO (medium-low PV)

WT vs. Mecp2 KO (low PV)

WT vs. Mecp2 KO

WT vs. Mecp2 KO

WT vs. Mecp2 KO

WT vs. Mecp2 KO

WT vs. Mecp2 KO

WT vs. Mecp2 KO

WT vs. Mecp2 KO

WT vs. Mecp2 KO

WT vs. Mecp2 KO

WT vs. Mecp2 KO

WT vs. Mecp2 KO

WT vs. Mecp2 KO

WT vs. Mecp2 KO

WT vs. Mecp2 KO

WT vs. Mecp2 KO

WT vs. Mecp2 KO

WT vs. Mecp2 KO

WT vs. Mecp2 KO

WT vs. Mecp2 KO

WT vs. Mecp2 KO

WT vs. Mecp2 KO

WT vs. Mecp2 KO

WT vs. Mecp2 KO

WT vs. Mecp2 KO

WT vs. Mecp2 KO

WT vs. Mecp2 KO (high PV)

WT vs. Mecp2 KO (medium-high PV)

WT vs. Mecp2 KO (medium-low PV)

WT vs. Mecp2 KO (low PV)

WT vs. Mecp2 KO

WT vs. Mecp2 KO

WT vs. Mecp2 KO

WT vs. Mecp2 KO (high PV)

WT vs. Mecp2 KO (medium-high PV)

WT vs. Mecp2 KO (medium-low PV)

WT vs. Mecp2 KO (low PV)

Genotype vs. RR

WT AC vs. Mecp2 KO-AC

WT AC vs. WT RR

KO-AC vs. KO-RR

Genotype vs. RR (medium-high PV)

Genotype vs. RR (high PV)

WT and Mecp2 KO

P56

Genotype vs. RR

P56

Genotype vs. RR

P56

Genotype vs. RR

P56

WT vs. Mecp2 Het

(Continued)
$P$ value

$t_{(10)}=2.82, p=0.018$

$t_{(10)}=3.24, p=0.009$

$p<0.001$

$t_{(10)}=3.31, p=0.008$

$t_{(10)}=1.91, p=0.085$

$t_{(10)}=1.93, p=0.083$

$t_{(10)}=1.81, p=0.101$

$t_{(10)}=0.61, p=0.555$

$t_{(10)}=3.24, p=0.268$

$p=0.002$

$t_{(10)}=0.32, p=0.756$

$t_{(10)}=1.92, p=0.083$

$t_{(10)}=1.29, p=0.228$

$t_{(10)}=1.54, p=0.155$

$t_{(10)}=3.62, p=0.004$

$t_{(7)}=4.22, p=0.003$

$t_{(10)}=4.05, p=0.002$

$t_{(8)}=1.97, p=0.08$

$t_{(10)}=2.50, p=0.03$

$t_{(8)}=0.19, p=0.85$

$t_{(10)}=1.32, p=0.21$

$t_{(8)}=1.79, p=0.11$

$t_{(8)}=4.89, p=0.001$

$t_{(8)}=3.39, p=0.009$

$t_{(14)}=0.91, p=0.38$

$t_{(8)}=0.24, p=0.81$

$t_{(8)}=0.24, p=0.82$

$t_{(8)}=0.29, p=0.78$

$t_{(9)}=0.14, p=0.89$

$t_{(8)}=0.02, p=0.99$

Genotype $F_{(1,136)}=37.07, p<0.001$

Genotype $F_{(1,136)}=18.26, p<0.001$

$p<0.05$

Genotype $F_{(1,96)}=2.73, p=0.108$

Genotype $F_{(1,96)}=12.44, p=0.001$

$p<0.01$

$t_{(10)}=3.67, p=0.004$

$\mathrm{t}_{(10)}=1.74, p=0.112$

$p<0.001$

$t_{(10)}=2.45, p=0.034$

$t_{(10)}=0.81, p=0.439$

$t_{(10)}=0.98, p=0.349$

$t_{(10)}=0.46, p=0.657$

$t_{(6)}=1.43, p=0.202$

$t_{(6)}=0.98, p=0.366$

$p=0.023$

$t_{(6)}=0.92, p=0.391$

$t_{(6)}=0.49, p=0.645$

$t_{(6)}=2.20, p=0.070$

$t_{(6)}=1.64, p=0.152$

$\operatorname{RR} F_{(7,112)}=11.62, p<0.0001$

Genotype $F_{(1,16)}=11.75, p=0.003$

Interaction $F_{(7,112)}=1.12, p=0.356$

$p=0.0449$

$p=0.0015$

$p<0.001$

Interaction $F_{(1,18)}=6.19, p=0.023$

Interaction $F_{(1,18)}=10.11, p=0.005$

Genotype $F_{(1,18)}=20.06, p=0.0003$

$\mathrm{RR} F_{(1,18)}=5.93, p=0.026$

$r:-0.653, p=0.006$

Genotype $F_{(1,16)}=117.91, p<0.001$ $\operatorname{RR} F_{(1,16)}=11.25, p=0.004$

Genotype $F_{(1,18)}=37.20, p<0.001$

$\operatorname{RR} F_{(1,18)}=27.33, p<0.001$

Genotype $F_{(1,16)}=43.89, p<0.001$

$\operatorname{RR} F_{(1,16)}=17.78, p=0.0007$

$p=0.8219$ 
Table 1. Continued

\begin{tabular}{|c|c|c|c|c|c|}
\hline Figure & Measurement & Type of test & Comparison & Age & $P$ value \\
\hline Fig. $8 E$ & Relative frequency of PV INs & Mann-Whitney $U$ test & WT vs. Mecp2 Het & $4 \mathrm{M}$ & $p=0.9798$ \\
\hline \multirow[t]{4}{*}{ Fig. $8 F$} & Fraction of PV INs & Unpaired $t$ test & WT vs. Mecp2 Het (high PV) & $4 \mathrm{M}$ & $t_{(10)}=1.51, p=0.163$ \\
\hline & & & WT vs. Mecp2 Het (medium-high PV) & $4 \mathrm{M}$ & $t_{(10)}=3.19, p=0.009$ \\
\hline & & & WT vs. Mecp2 Het (medium-low PV) & $4 \mathrm{M}$ & $t_{(10)}=1.03, p=0.327$ \\
\hline & & & WT vs. Mecp2 Het (low PV) & $4 \mathrm{M}$ & $t_{(10)}=0.77, p=0.456$ \\
\hline Fig. $8 \mathrm{H}$ & Relative frequency of PV INs & Mann-Whitney $U$ test & WT vs. Mecp2 Het & $8 \mathrm{M}$ & $p<0.001$ \\
\hline \multirow[t]{4}{*}{ Fig. 81} & Fraction of PV INs & Unpaired $t$ test & WT vs. Mecp2 Het (high PV) & $8 \mathrm{M}$ & $t_{(10)}=3.16, p=0.013$ \\
\hline & & & WT vs. Mecp2 Het (medium-high PV) & $8 \mathrm{M}$ & $t_{(10)}=0.52, p=0.620$ \\
\hline & & & WT vs. Mecp2 Het (medium-low PV) & $8 \mathrm{M}$ & $t_{(10)}=4.06, p=0.004$ \\
\hline & & & WT vs. Mecp2 Het (low PV) & $8 \mathrm{M}$ & $t_{(10)}=1.40, p=0.200$ \\
\hline Fig. 8J & Rotarod task & Two-way ANOVA & Genotype vs. RR & $8 \mathrm{M}$ & $\begin{array}{l}\operatorname{RR} F_{(7,176)}=23.60, p<0.001 \\
\text { Genotype } F_{(1,176)}=42.81, p<0.001 \\
\text { Interaction } F_{(7,176)}=0.66, p=0.706\end{array}$ \\
\hline Fig. $8 \mathrm{~J}$ & Correlation between \% HIGH PV and RR performance & Pearson's $r$ & WT and Mecp2 Het & $8 \mathrm{M}$ & $r:-0.481, p=0.024$ \\
\hline
\end{tabular}

evaluated the activation of these cells in the $S 1$ cortex by assessing the levels of PV immunofluorescence, which correlates with both activity and plasticity (Volman et al., 2011; Donato et al., 2013; Fig. 1A, F). The analysis of $\mathrm{S} 1$ cortices from fully symptomatic mice at P56 (De Filippis et al., 2010) confirmed previous studies (Tomassy et al., 2014) showing a significant increase in the density of $\mathrm{PV}^{+}$ cells in the upper layers of the cortex in Mecp2 KO mice compared to WT animals (Fig. 1B). Moreover, mean fluorescence intensity (Fig. 1C) and cumulative frequency distribution analyses (Fig. 1D) indicated that PV intensity showed higher values in Mecp2 KO mice compared to WT littermates. Using arbitrary criteria to differentiate $\mathrm{PV}^{+} \mathrm{INs}$ in four subclasses based on fluorescence intensity (see Materials and Methods), we found that in Mecp2 KO mice there was a robust increase in the percentage of the high-expressing fraction compared to WT animals (Fig. $1 E$ ). These results were confirmed by the analysis of the absolute density of cells, showing that Mecp2 loss affects, exclusively, the high-PV subclass (high-PV, WT: $28.55 \mathrm{cells} / \mathrm{mm}^{2} \pm 12.19$, Mecp2 KO: $87.08 \mathrm{cells} / \mathrm{mm}^{2} \pm$ 9.50. $t$ test $\left.t_{(10)}=3.79, p=0.004 ; n=6\right)$.

In contrast, there were no significant differences between P28 presymptomatic Mecp2 KO mice and WT littermates in either the density of $\mathrm{PV}^{+}$INs in layer II/III of the cortex (Fig. 1G) or the mean fluorescence intensity of PV (Fig. 1H). In addition, while cumulative frequency distribution analysis showed a very modest, although statistically significant, rightward PV intensity shift in mutant mice compared to WT (Fig. 1/), the analysis of $\mathrm{PV}^{+} \mathrm{IN}$ subclasses revealed no significant differences between genotypes at P28 (Fig. 1J). Taken together, these results suggest that PV network is shifted toward a high levels of $\mathrm{PV}$ expression in the S1 cortex of Mecp2 KO mice, an alteration that appears when the pathologic signs are established.

\section{Early-onset supernumerary excitatory connectivity onto $\mathrm{PV}^{+}$INs in Mecp2 KO mice}

To assess possible mechanisms underlying the altered PV-configuration in Mecp2 mutant mice, we analyzed excitatory/inhibitory (E/l) input connectivity of identified $\mathrm{PV}^{+}$INs inspecting $\mathrm{S} 1$ sections using double immunofluorescence and confocal microscopy. Excitatory presynaptic terminals were identified with antibodies for the vesicular glutamate transporter (VGLUT1), while inhibitory presynaptic terminals were identified using antibodies for vesicular GABA transporter (VGAT; Fig. 2Q).

In symptomatic Mecp2 KO mice at P56, the density of VGLUT1 ${ }^{+}$puncta on $\mathrm{PV}^{+}$dendrites and somata in layer II/III was significantly higher compared to age-matched WT mice (Fig. 2A, C, E, G). To exclude that higher density of VGLUT1 $1^{+}$puncta is secondary to the smaller size of $\mathrm{PV}^{+}$INs shown by these mutants (Tomassy et al., 2014), we compared the mean number of puncta decorating individual somata in each genotype. This analysis showed that Mecp2 KO mice are contacted by a significant higher number of VGLUT1 ${ }^{+}$puncta per $\mathrm{PV}^{+}$cell body than WT mice (WT $7.58 \pm 0.39$ boutons/soma, Mecp2 KO $8.63 \pm$ 0.18 ; $t$ test $t(10)=2.396, p=0.037 ; n=6)$. Moreover, we found that the density of $\mathrm{VGAT}^{+}$puncta on $\mathrm{PV}^{+}$dendrites was significantly lower in Mecp2 KO mice compared to WT mice (Fig. 2B, $F$ ), while somata inhibitory innervation was not affected (Fig. 2D, H). Mecp2 KO mice at P56 showed higher density of excitatory VGLUT $1^{+}$presynaptic terminals on $\mathrm{CR}^{+}$dendrites (Fig. 2l, M), but not on the somata (Fig. $2 K, O$ ). In contrast, the densities of $\mathrm{VGAT}^{+}$ terminals on both dendrites and somata of $\mathrm{CR}^{+}$INs were not statistically different between genotypes (Fig. $2 J, N, L$, $P$ ). These observations indicate that in symptomatic Mecp2 KO there is a shift toward increased excitatory synaptic innervation in both $\mathrm{PV}^{+}$and $\mathrm{CR}^{+} \mathrm{INs}$, with $\mathrm{PV}^{+}$ INs showing a higher extent of altered E/I connectivity consistent with higher PV expression in these cells (Donato et al., 2013).

In presymptomatic Mecp2 KO mice, the density of VGLUT1-labeled puncta decorating $\mathrm{PV}^{+}$dendrites in layer II/III was significantly higher compared to age-matched WT mice (Fig. $3 A, E$ ), while the number of excitatory inputs on the somata was not affected (Fig. 3C, G). In contrast, the density of $\mathrm{VGAT}^{+}$puncta on $\mathrm{PV}^{+}$dendrites was significantly lower in Mecp2 KO mice (Fig. 3B, F), while inhibitory innervation on $\mathrm{PV}^{+}$somata was unaffected (Fig. $3 D, H)$. Interestingly, the density of VGLUT1 ${ }^{+}$and VGAT ${ }^{+}$ puncta onto both $\mathrm{CR}^{+}$soma and dendrites in the $\mathrm{S} 1$ cortex were comparable between Mecp2 KO and WT mice at P28 (Fig. 3/-P). These data indicate that selective alterations in E/I input balance of INs in S1 cortex of presymptomatic Mecp2 $\mathrm{KO}$ animals are specific for the 
Table 2. Data structure (normal or non-normal distribution), statistical tests, and observed power value of the statistical test

\begin{tabular}{|c|c|c|c|}
\hline Figure & Data structure & Type of test & Power \\
\hline Fig. $1 B$ & Normal distribution & Unpaired $t$ test & 0.720 \\
\hline Fig. $1 C$ & Normal distribution & Unpaired $t$ test & 0.757 \\
\hline Fig. $1 E$ & Normal distribution & Unpaired $t$ test & Low 0.372 ; medium-low 0.413 ; medium-high 0.410 ; high 0.845 \\
\hline Fig. $1 \mathrm{G}$ & Normal distribution & Unpaired $t$ test & 0.086 \\
\hline Fig. $1 H$ & Normal distribution & Unpaired $t$ test & 0.186 \\
\hline Fig. $1 \mathrm{~J}$ & Normal distribution & Unpaired $t$ test & Low 0.286 ; medium-low 0.214 ; medium-high 0.412 ; high 0.059 \\
\hline Fig. $2 E$ & Normal distribution & Unpaired $t$ test & 0.902 \\
\hline Fig. $2 F$ & Normal distribution & Unpaired $t$ test & 0.823 \\
\hline Fig. $2 G$ & Normal distribution & Unpaired $t$ test & 0.953 \\
\hline Fig. $2 H$ & Normal distribution & Unpaired $t$ test & 0.410 \\
\hline Fig. $2 M$ & Normal distribution & Unpaired $t$ test & 0.616 \\
\hline Fig. $2 N$ & Normal distribution & Unpaired $t$ test & 0.053 \\
\hline Fig. 20 & Normal distribution & Unpaired $t$ test & 0.232 \\
\hline Fig. $2 P$ & Normal distribution & Unpaired $t$ test & 0.352 \\
\hline Fig. $3 E$ & Normal distribution & Unpaired $t$ test & 0.989 \\
\hline Fig. $3 F$ & Normal distribution & Unpaired $t$ test & 0.837 \\
\hline Fig. $3 G$ & Normal distribution & Unpaired $t$ test & 0.136 \\
\hline Fig. $3 H$ & Normal distribution & Unpaired $t$ test & 0.055 \\
\hline Fig. $3 M$ & Normal distribution & Unpaired $t$ test & 0.055 \\
\hline Fig. $3 N$ & Normal distribution & Unpaired $t$ test & 0.057 \\
\hline Fig. 30 & Normal distribution & Unpaired $t$ test & 0.052 \\
\hline Fig. $3 P$ & Normal distribution & Unpaired $t$ test & 0.050 \\
\hline Fig. $4 C$ & Normal distribution & Two-way ANOVA with RM & Genotype 0.978 \\
\hline Fig. $4 D$ & Normal distribution & Two-way ANOVA with RM & Genotype 0.729 \\
\hline Fig. $4 G$ & Normal distribution & Two-way ANOVA with RM & Genotype 1.000 \\
\hline Fig. $4 H$ & Normal distribution & Two-way ANOVA with RM & Genotype 0.856 \\
\hline Fig. $5 B$ & Normal distribution & Unpaired $t$ test & 0.910 \\
\hline Fig. $5 C$ & Normal distribution & Unpaired $t$ test & 0.053 \\
\hline Fig. $5 E$ & Normal distribution & Unpaired $t$ test & Low 0.070; medium-low 0.145; medium-high 0.206; high 0.599 \\
\hline Fig. $5 G$ & Normal distribution & Unpaired $t$ test & 0.228 \\
\hline Fig. $5 H$ & Normal distribution & Unpaired $t$ test & 0.132 \\
\hline Fig. $5 J$ & Normal distribution & Unpaired $t$ test & Low 0.283; medium-low 0.456; medium-high 0.070 ; high 0.123 \\
\hline Fig. $6 A$ & Normal distribution & Two-way ANOVA with RM & Interaction 0.464 ; test $0.957 ;$ genotype 0.895 \\
\hline Fig. $6 D$ & Normal distribution & Two-way ANOVA & $\begin{array}{l}\text { (Low) interaction } 0.666 \text {; test } 0.094 \text {; genotype } 0.228 \\
\text { (Medium-low) interaction } 0.113 \text {; test } 0.278 \text {; genotype } 0.919 \\
\text { (Medium-high) interaction } 0.739 \text {; test } 0.466 \text {; genotype } 0.524 \\
\text { (High) interaction } 0.914 \text {; test } 0.721 \text {; genotype } 0.997\end{array}$ \\
\hline Fig. $6 E$ & Normal distribution & Pearson's $r$ & 0.829 \\
\hline Fig. $7 B$ & Normal distribution & Two-way ANOVA & Interaction 0.996 ; test 0.940 ; genotype 1.000 \\
\hline Fig. $7 C$ & Normal distribution & Two-way ANOVA & Interaction 0.152 ; test 0.999 ; genotype 0.999 \\
\hline Fig. $7 D$ & Normal distribution & Two-way ANOVA & Interaction 0.999; test 0.999 ; genotype 0.993 \\
\hline Fig. $7 E$ & Normal distribution & Two-way ANOVA & Interaction 0.209 ; test 0.723 ; genotype 0.290 \\
\hline Fig. $8 \mathrm{C}$ & Normal distribution & Unpaired $t$ test & $\begin{array}{l}\text { Low } 0.062 ; \text { medium-low } 0.536 \\
\text { Medium-high } 0.310 ; \text { high } 0.081\end{array}$ \\
\hline Fig. $8 F$ & Normal distribution & Unpaired $t$ test & $\begin{array}{l}\text { Low 0.108; medium-low } 0.154 \\
\text { Medium-high 0.820; High } 0.276\end{array}$ \\
\hline Fig. 8I & Normal distribution & Unpaired $t$ test & $\begin{array}{l}\text { Low } 0.234 ; \text { medium-low } 0.942 \\
\text { Medium-high } 0.074 ; \text { high } 0.791\end{array}$ \\
\hline Fig. 8J & Normal distribution & Two-way ANOVA with RM & Interaction 0.370 ; test 1.000 ; genotype 0.844 \\
\hline Fig. $8 K$ & Normal distribution & Pearson's $r$ & 0.647 \\
\hline
\end{tabular}

subpopulation of $\mathrm{PV}^{+}$INs and precede the shift toward high-PV expression in this cortical network.

\section{Early-onset network activity reduction in S1 cortical slices of Mecp2 KO mice}

To assess the consequences of increased weight of excitation reaching INs in Mecp2 KO mice on S1 cortex activity, we measured voltage-sensitive dye (VSD) signals evoked by stimulation of intracortical afferents in acute S1 slices from symptomatic (P45-P60) and presymptomatic (P24-P26) Mecp2 KO and age-matched WT mice. The amplitude of VSD signals $(\Delta F / F)$ evoked in layer II/III by afferent stimulation in layer $\mathrm{V}$ was proportional to the amplitude of fEPSPs at different afferent stimulation intensities and followed the same kinetic profile (Fig. 4A). The amplitude of VSD signals were significantly smaller in $\mathrm{S} 1$ slices from symptomatic Mecp2 KO mice compared to those from WT mice at all stimulus intensities except the lowest (Fig. 4C). In addition, the spatial spread and spatiotemporal profiles of VSD signals through layer I-V was significantly smaller in Mecp2 KO slices than in WT slices, also at all stimulus intensities except the lowest (Fig. 4B, 


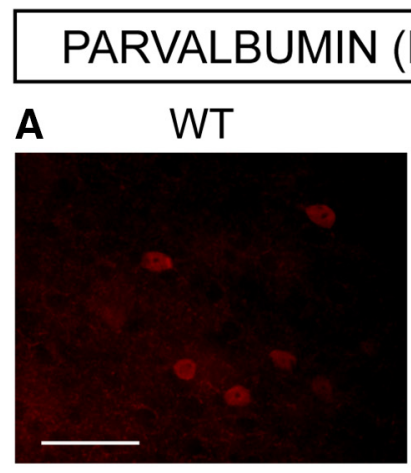

B

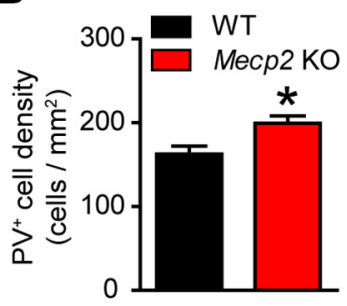

C

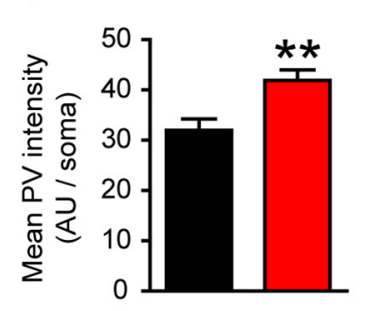

D

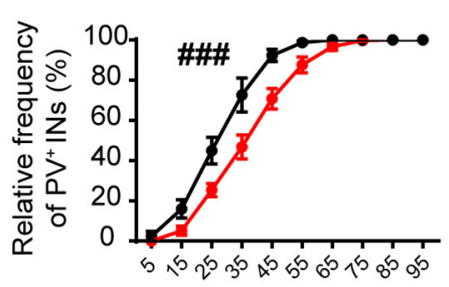

PV intensity

E

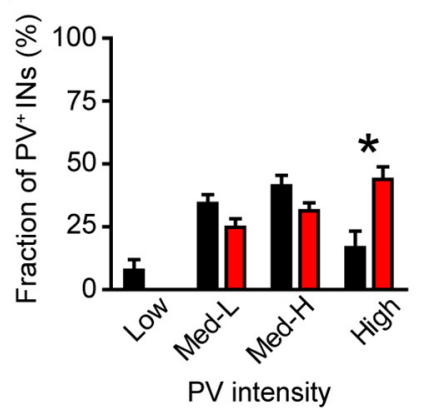

PARVALBUMIN (P28, S1 Layer II/III)

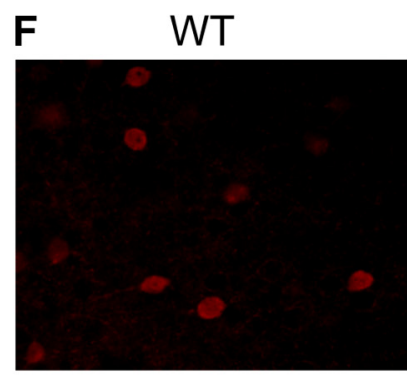

G

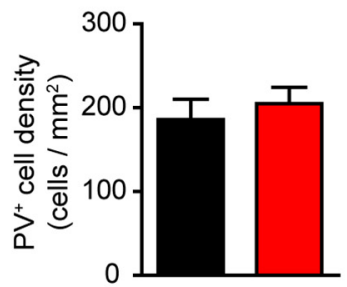

I

H

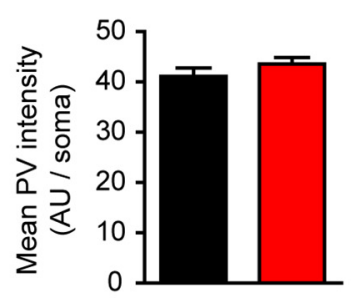

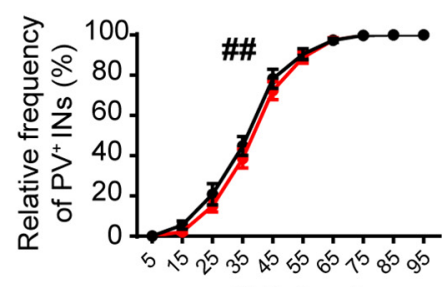

PV intensity

Mecp2 KO

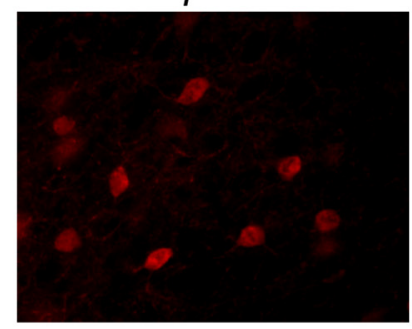

J

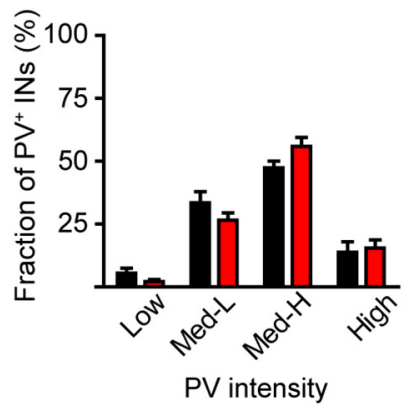

Figure 1. Atypical high-PV expression in the S1 cortex of Mecp2 KO mice. A, Representative images showing PV labeling in layer II/III of S1 cortex in WT and Mecp2 KO mice at P56. Histograms showing quantitative analysis of $\mathrm{PV}^{+}$cell density $(\boldsymbol{B})$, PV mean fluorescence intensity $(\boldsymbol{C})$, cumulative $(\boldsymbol{D})$ and binned $(\boldsymbol{E})$ frequency distribution of PV cells intensity in WT and Mecp2 KO mice at P56. $\boldsymbol{F}$, Representative images showing PV labeling in layer II/III of S1 cortex in WT and Mecp2 KO mice at P28. Histograms showing quantitative analysis of PV cell density $(\boldsymbol{G})$, PV mean fluorescence intensity $(\boldsymbol{H})$, cumulative $(\boldsymbol{I})$ and binned $(\boldsymbol{J})$ frequency distribution of PV cells intensity in WT and Mecp2 KO mice at P28. $n=6$ mice per genotype. Student's $t$ test: $* p<0.05$, $* * p<0.01$, Mann-Whitney $U$ test for $\boldsymbol{D}, \boldsymbol{I}:{ }^{\# \#} p<0.01 ;{ }^{\# \# \#} p<0.001$. Scale bar $=100 \mu \mathrm{m}$.

$D$, E). When we analyzed presymptomatic Mecp2 KO mice, we found a trend toward decreased maximum amplitudes of VSD signals in S1 slices from mutant mice compared with WT slices which did not reach statistical significance (Fig. 4G). Moreover, as in older mice, the spatial spread and spatio-temporal profile of VSD signals through layer I-V was significantly smaller in mutant slices than in WT controls at all stimulus intensities (Fig. 4F, H, I). These results demonstrate that, consistent with an E/I imbalance favoring synaptic inhibition, the $\mathrm{S} 1$ cortex of Mecp2 KO mice is hypoactive in response to layer $\mathrm{V}$ stimulation before overt pathologic phenotypes.

MeCP2 loss disrupts both basal and experiencedependent changes of PV expression in M1 cortex of behaviorally impaired Mecp2 KO mice

Although structural alterations affecting pyramidal excitatory neurons in the M1 cortex of both RTT individuals and Mecp2 KO mice were reported (Armstrong et al., 1998; Belichenko et al., 2009), whether the organization of $\mathrm{PV}^{+}$INs network in this area is affected it is still unknown. Similar to what we observed in S1, P56 Mecp2 KO mice (Fig. 5A) show a significant higher density of $\mathrm{PV}^{+}$INs in layer II/III of the M1 cortex than WT mice (Fig. 5B). Moreover, while mean fluorescence intensity of PV was unaffected in $\mathrm{M} 1$ of symptomatic mutants (Fig. 5C), cumulative frequency distribution analysis showed a shift toward higher PV levels in $\mathrm{PV}^{+}$neurons (Fig. $5 D$ ), a finding that was corroborated by the significant increase of the percentage of the high-PV fraction compared with WT animals (Fig. $5 E$ ), which is consistent with our observations in the S1 cortex. These results were confirmed by the analysis of the absolute density of cells, showing that Mecp2 loss affects, exclusively, the high-PV subclass in M1 cortex (high-PV, WT: 9.98 cells $/ \mathrm{mm}^{2} \pm 2.86$, Mecp2 KO: 30.44 cells $/ \mathrm{mm}^{2} \pm 5.56$. $t$ test $t_{(10)}=3.27, p=0.008 ; n=6$ ). 
PARVALBUMIN (P56, S1 Layer II/III)

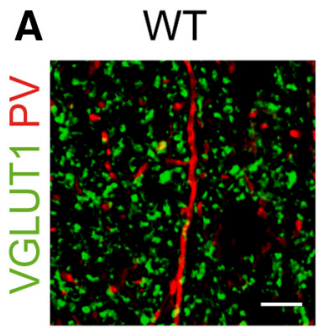

B

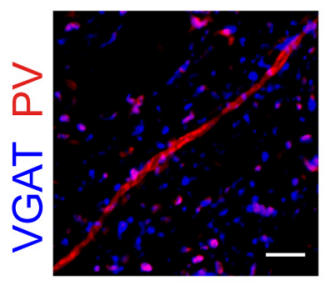

C

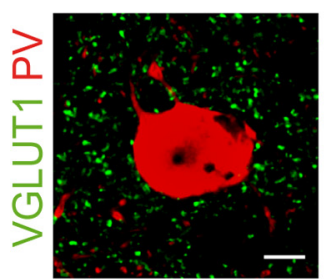

D

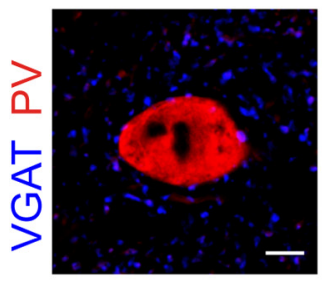

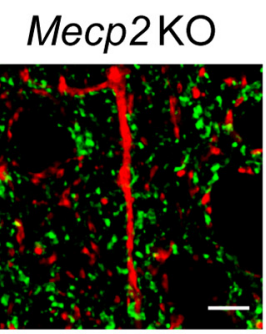

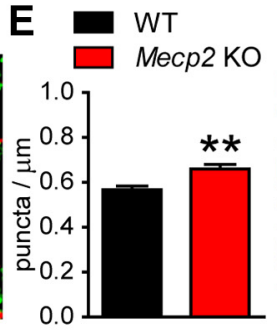

$\mathbf{F}$
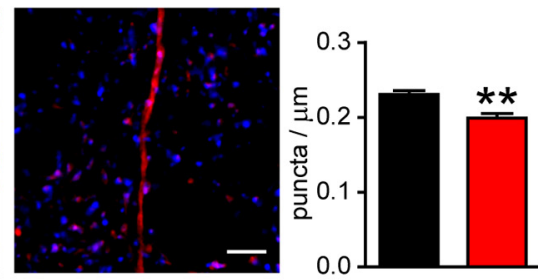

G
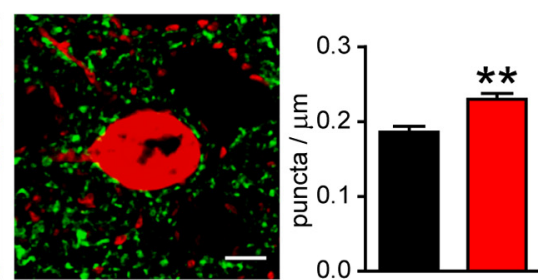

H

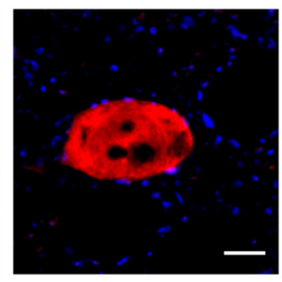

\section{CALRETININ (P56, S1 Layer II/III)}

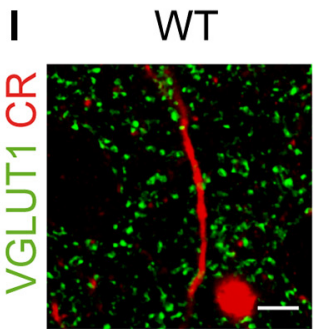

J

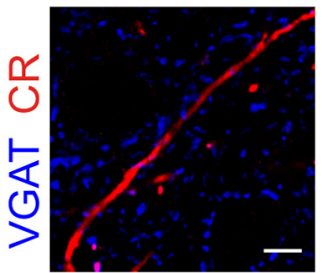

$\mathrm{K}$
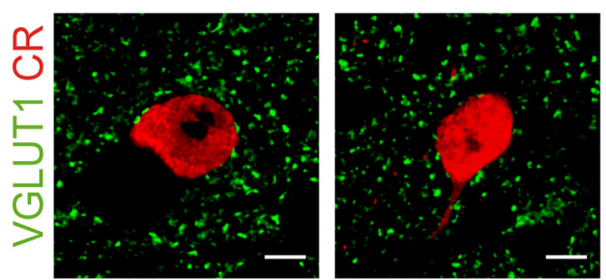

$\mathbf{L}$

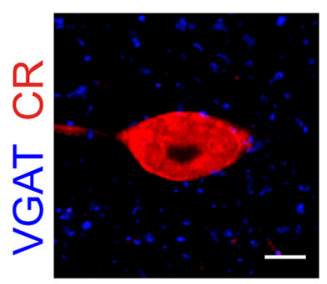

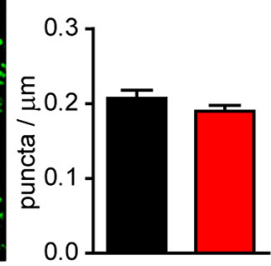

\section{Mecp2KO M}

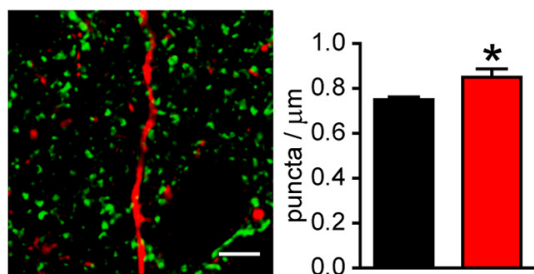

N
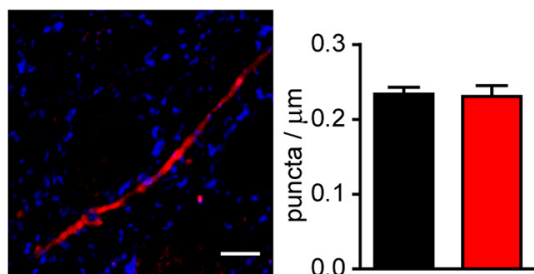

0
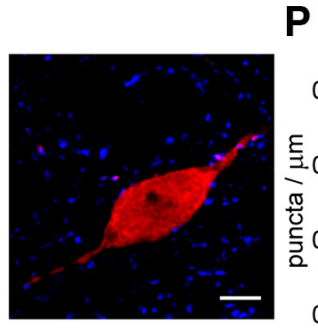

Q
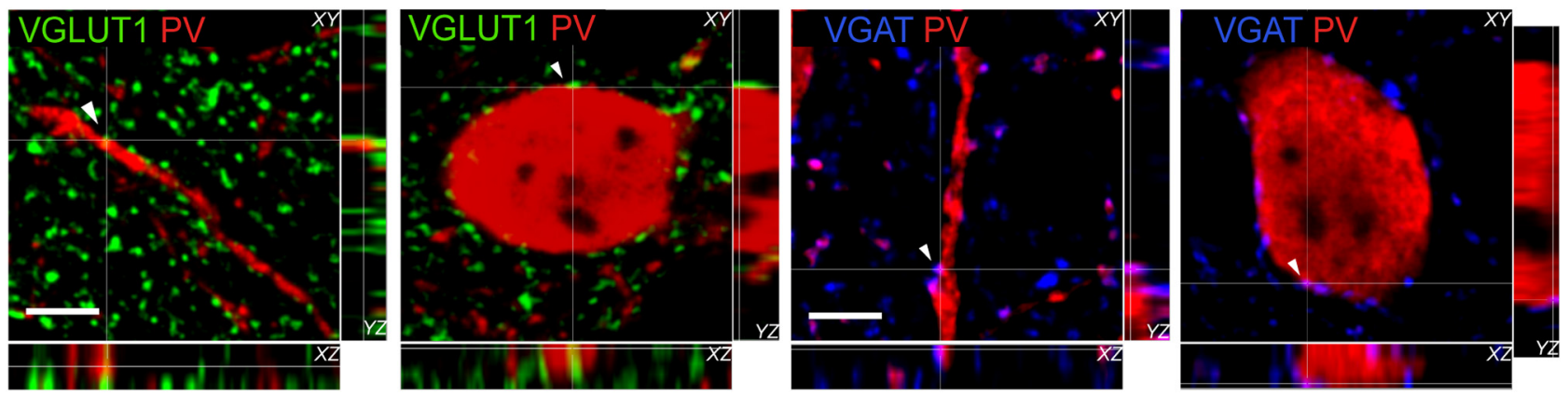

Figure 2. Distribution of excitatory and inhibitory presynaptic terminals onto $\mathrm{PV}^{+}$and $\mathrm{CR}^{+} \mathrm{INs}$ in $\mathrm{P} 56 \mathrm{Mecp} 2 \mathrm{KO}$ mice. Representative confocal images of $\mathrm{VGLUT1}^{+}$(green: $\boldsymbol{A}, \boldsymbol{C}$ ) and $\mathrm{VGAT}^{+}$(blue: $\boldsymbol{B}, \boldsymbol{D}$ ) puncta corresponding to excitatory and inhibitory presynaptic terminals, respectively, apposed to dendrites (top) and somata (bottom) of PV ${ }^{+}$INs in layer II/III of S1 cortex in P56 WT and Mecp2 $\mathrm{KO}$ mice. Histograms showing quantitative analysis in WT and Mecp2 KO mice of VGLUT1 ${ }^{+}$and VGAT ${ }^{+}$puncta density contacting either dendrites $(\boldsymbol{E}, \boldsymbol{F})$ or somata $(\boldsymbol{G}, \boldsymbol{H})$, respectively, of $\mathrm{PV}^{+}$INs. Confocal images showing VGLUT1 ${ }^{+}($green: $\boldsymbol{I}, \boldsymbol{K})$ and VGAT ${ }^{+}($blue: $\boldsymbol{J}, \boldsymbol{L}$ ) puncta contacting dendrites (top) and somata (bottom) of $\mathrm{CR}^{+}$INs in layer II/III of S1 cortex in WT and Mecp2 KO mice. Histograms showing quantitative analysis in WT and Mecp2 KO mice of VGLUT1 ${ }^{+}$and $\mathrm{VGAT}^{+}$puncta density contacting either dendrites $(\boldsymbol{M}, \boldsymbol{N})$ or somata $(\boldsymbol{O}, \boldsymbol{P})$, respectively, of $\mathrm{CR}^{+}$INs. PV: VGLUT1 $n=6$ mice per genotype; VGAT dendrites $n=5$ WT and 4 Mecp2 KO mice per genotype; VGAT soma $n=5$ mice per genotype; CR: VGLUT1 $n=6$ mice and VGAT $n=5$ mice per genotype. 
continued

Student's $t$ test: $* p<0.05 ; * * p<0.01$. $Q$, Representative 3D projections in three image planes showing excitatory VGLUT1 ${ }^{+}$(green) and inhibitory $\mathrm{VGAT}^{+}$(blue) synaptic terminals contacting $\mathrm{PV}^{+}$cell bodies and dendrites (red). Arrowheads point to selected $\mathrm{VGLUT1}^{+}$and VGAT ${ }^{+}$puncta apposed to dendrites or somata of $\mathrm{PV}^{+}$interneurons at the intersection of the XY cross. Note the lack of black pixels between the presynaptic puncta and the postsynaptic structures. Scale bars $=5 \mu \mathrm{m}$.

As in S1 cortex, in M1 of P28 mice (Fig. 5F) neither the density of $\mathrm{PV}^{+}$INs (Fig. $5 \mathrm{G}$ ) nor the mean fluorescence intensity (Fig. $5 H$ ) were different between genotypes. Whereas cumulative frequency distribution analysis showed a very modest, although statistically significant, rightward PV intensity shift in mutant mice compared with WT (Fig. 5/), the distribution of $\mathrm{PV}^{+}$INs subclasses in M1 revealed no differences between genotypes (Fig. 5J). These results indicate that the progression of the pathology in Mecp2 KO mice is associated with an increased

\section{PARVALBUMIN (P28, S1 Layer II/III)}

\section{CALRETININ (P28, S1 Layer II/III)}
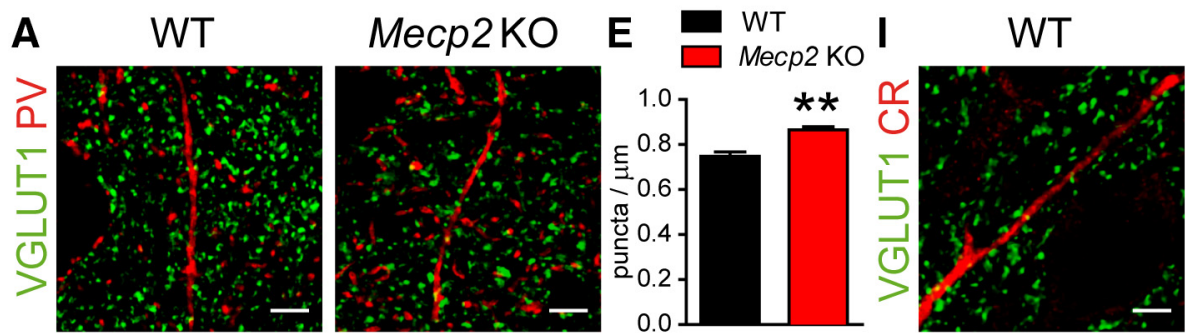

Mecp2 KO M

B

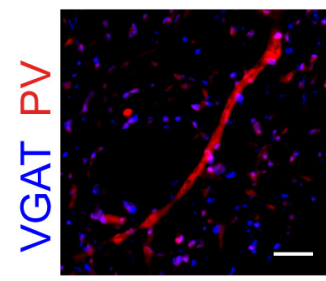

C

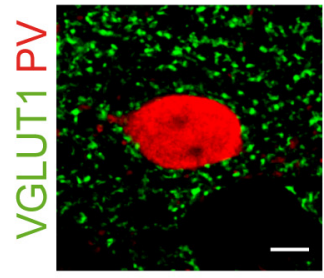

D

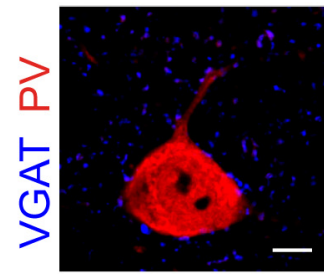

$\mathbf{F}$

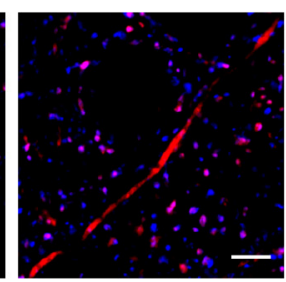

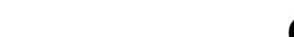

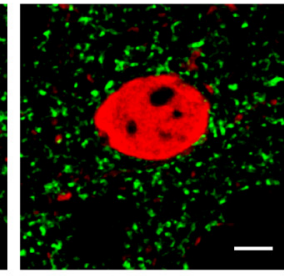

G

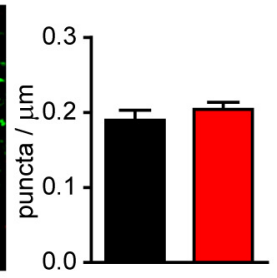

$\mathbf{H}$
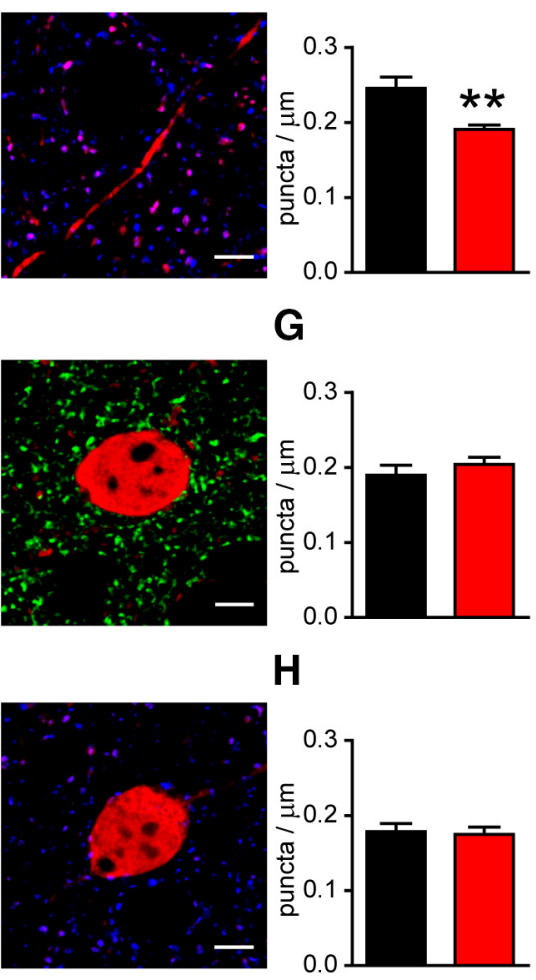

J

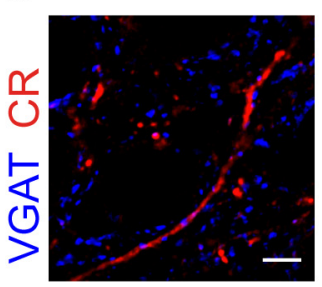

K

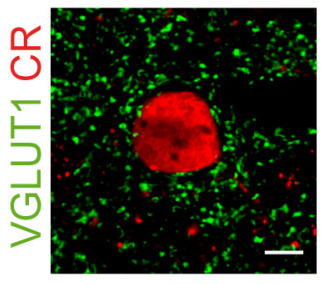

$\mathbf{L}$

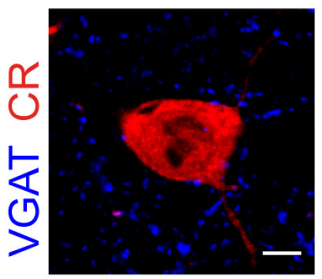

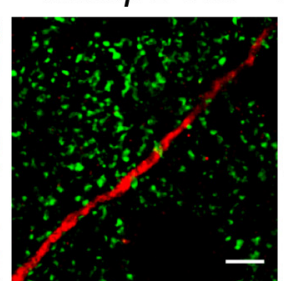

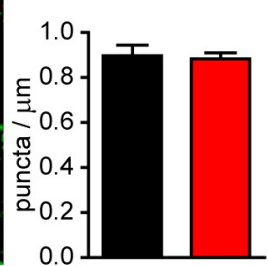

$\mathbf{N}$
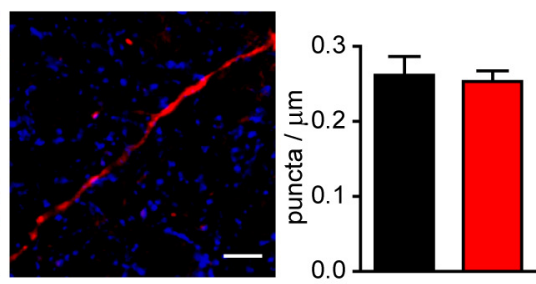

0
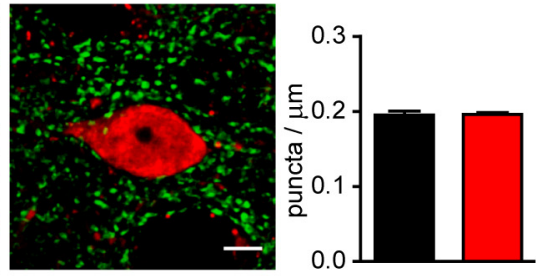

$\mathbf{P}$

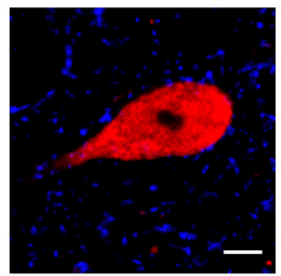

Figure 3. Distribution of excitatory and inhibitory presynaptic terminals onto $\mathrm{PV}^{+}$INs in P28 Mecp2 KO mice. Representative confocal images of excitatory, VGLUT1 ${ }^{+}$(green: $\boldsymbol{A}, \boldsymbol{C}$ ) and inhibitory, $\mathrm{VGAT}^{+}$(blue: $\boldsymbol{B}, \boldsymbol{D}$ ) synaptic terminals contacting $\mathrm{PV}^{+}$(red) dendrites (top) and somata (bottom) in layer II/III of S1 cortex in P28 WT and Mecp2 KO mice. Histograms showing quantitative analysis in WT and Mecp2 KO mice of VGLUT1 ${ }^{+}$and VGAT ${ }^{+}$puncta density contacting either dendrites $(\boldsymbol{E}, \boldsymbol{F})$ or somata $(\boldsymbol{G}, \boldsymbol{H})$, respectively, of PV ${ }^{+}$INs. Representative confocal images of $\mathrm{VGLUT1}^{+}$(green: $\boldsymbol{I}, \boldsymbol{K}$ ) and $\mathrm{VGAT}^{+}$(blue: $\boldsymbol{J}, \boldsymbol{L}$ ) puncta contacting $\mathrm{CR}^{+}$(red) dendrites (top) and somata (bottom) in layer II/III of S1 cortex in P28 Mecp2 KO mice and WT littermates. Histograms showing quantitative analysis in WT and Mecp2 KO mice of VGLUT1 ${ }^{+}$and VGAT ${ }^{+}$puncta density contacting either dendrites $(\boldsymbol{M}, \boldsymbol{N})$ or somata $(\boldsymbol{O}, \boldsymbol{P})$, respectively, of $\mathrm{CR}^{+}$ INs. PV: GLUT1 dendrites $n=5$ mice per genotype; GLUT1 soma $n=8$ mice per genotype; VGAT $n=5$ mice per genotype; CR: VGLUT1 and VGAT $n=5$ mice per genotype. Student's $t$ test: $* * p<0.01$. Scale bars $=5 \mu \mathrm{m}$. 
A
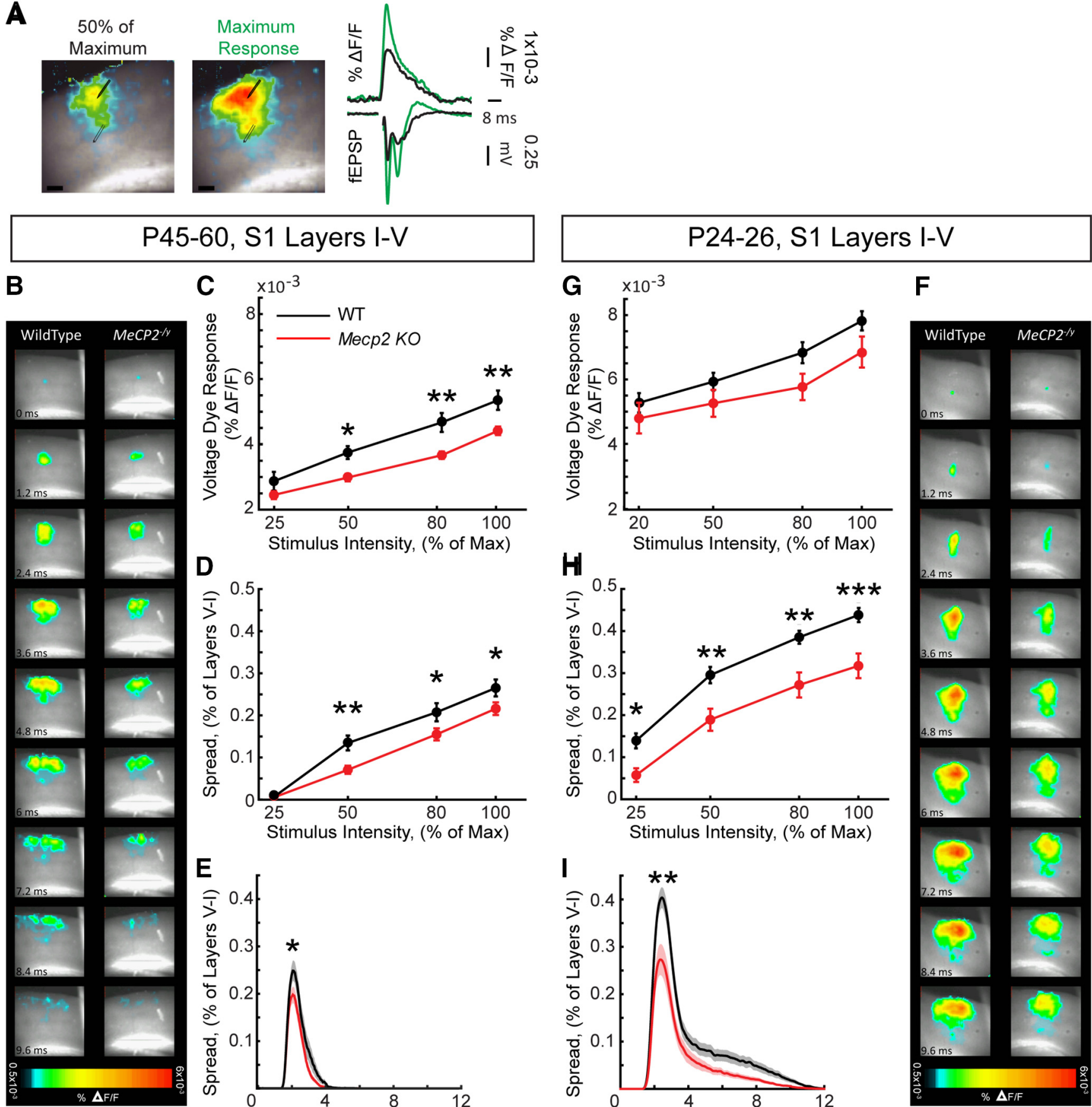

$\mathbf{F}$
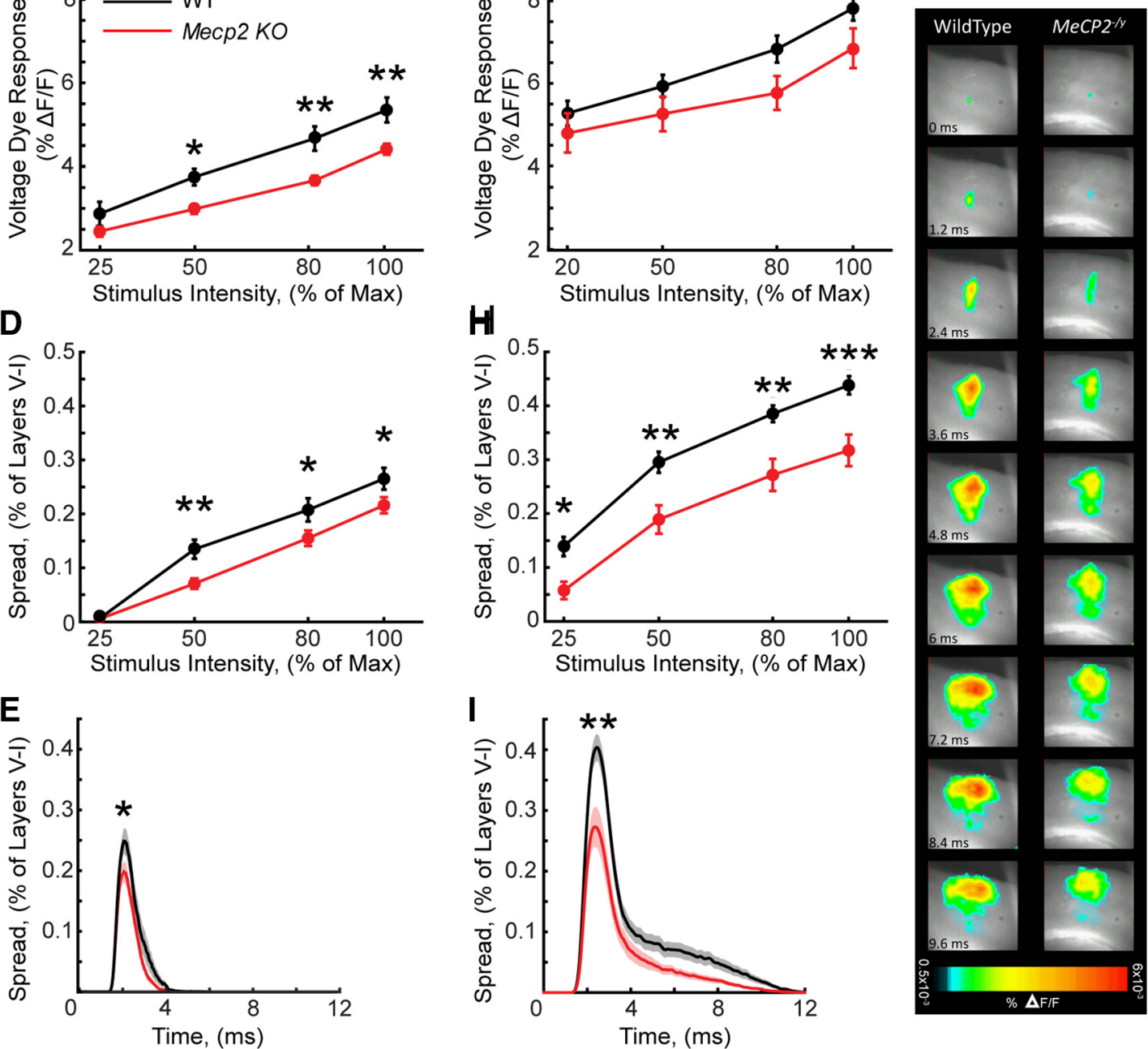

Figure 4. Smaller amplitude and spatial spread of synaptically induced neuronal depolarizations in layer II/III of S1 cortex in presymptomatic and symptomatic Mecp2 KO mice. A, Representative example (left) of a VSD-stained S1 slice with superimposed evoked VSD signals expressed as $\Delta F / F$, and displayed in a pseudo-color scale (warmer colors represent larger VSD amplitudes). Representative examples (right) of fEPSPs and VSD $\Delta F / F$ traces at lower (50\% maximum response) and higher (maximum response) stimulation intensities. $\boldsymbol{B}, \boldsymbol{F}$, Frames of representative time-lapse movies of VSD-stained slices during a single fEPSP in symptomatic $(\boldsymbol{B})$ and presymptomatic $(\boldsymbol{F})$ mice. $\boldsymbol{C}, \mathbf{G}$, Input-output relationship between afferent stimulus intensity and the amplitude of VSD signals expressed as \% $\Delta F / F$ in symptomatic $(\boldsymbol{C})$ and presymptomatic $(\boldsymbol{G})$ mice. $\boldsymbol{D}, \boldsymbol{H}$, Input-output relationship between afferent stimulus intensity and the spatial spread of signal through cortical layers I-V in symptomatic $(\boldsymbol{D})$ and presymptomatic $(\boldsymbol{H})$ mice. $\boldsymbol{E}$, $\boldsymbol{I}$, Spatio-temporal spread of VSD signals at maximum response stimulation in symptomatic $(\boldsymbol{E})$ and presymptomatic $(\boldsymbol{I})$ mice. Solid lines represent the mean; shaded areas represent the standard error of the mean. $n=12$ slices from 4 WT mice; $n=24$ slices from 6 Mecp2 $\mathrm{KO}$ mice at P45-P50; $n=17$ slices from 3 WT and Mecp2 KO mice at P24-P26. Two-way ANOVA and Bonferroni posthoc tests for $\boldsymbol{C}, \boldsymbol{D}, \boldsymbol{G}, \boldsymbol{H}$ and $t$ test of area under the curve for $\boldsymbol{E}, \boldsymbol{I} . * p<0.05, * * p<0.01, * * * p<0.001$. Scale bars $=100 \mu \mathrm{m}$. 


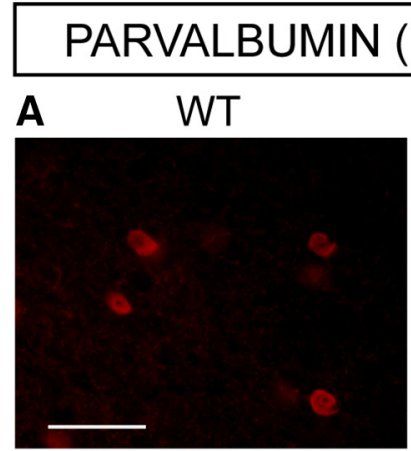

B

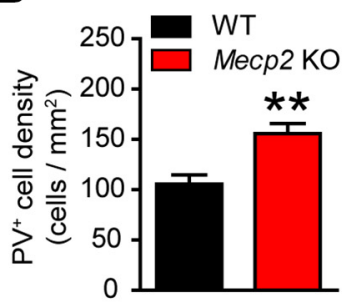

C

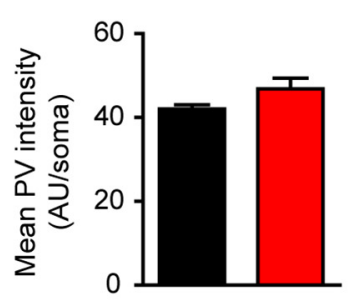

D

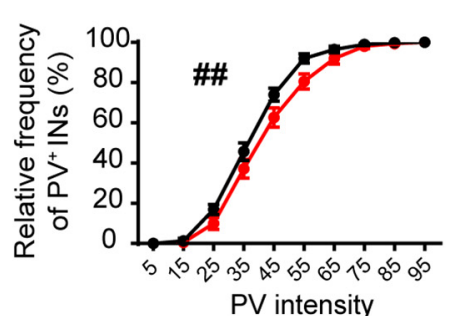

E

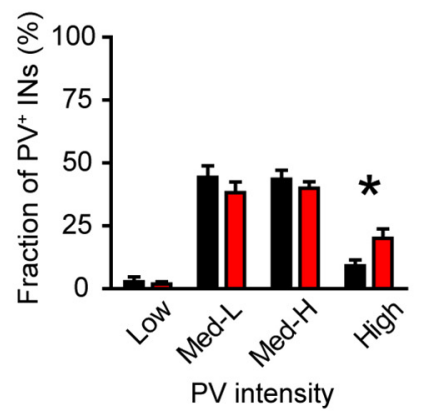

\section{PARVALBUMIN (P28, M1 Layer II/III)}

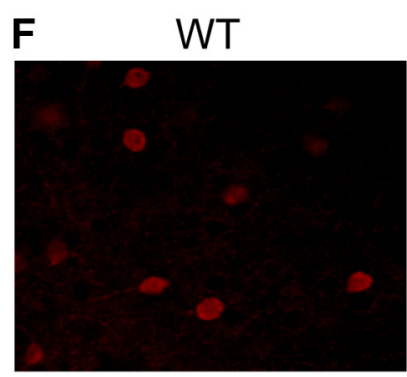

G

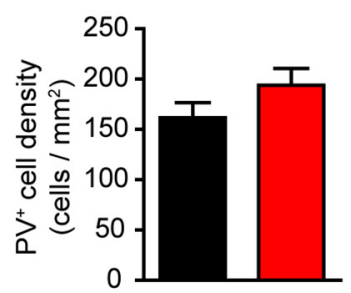

I

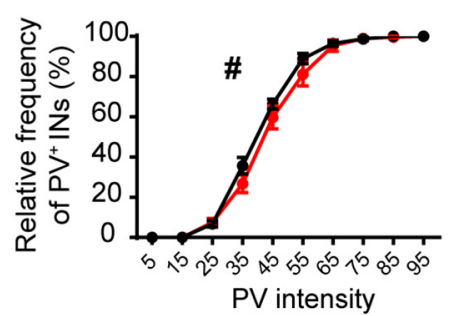

H

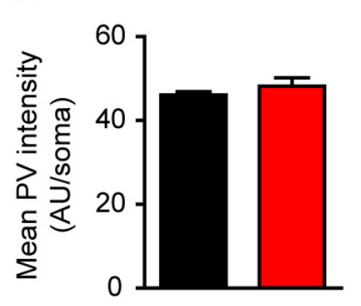

Mecp2 KO

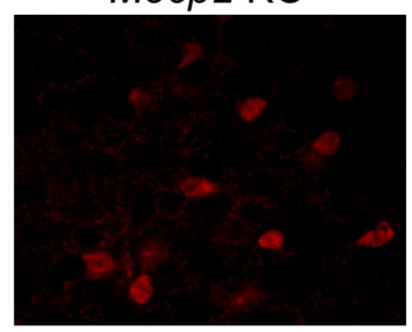

J

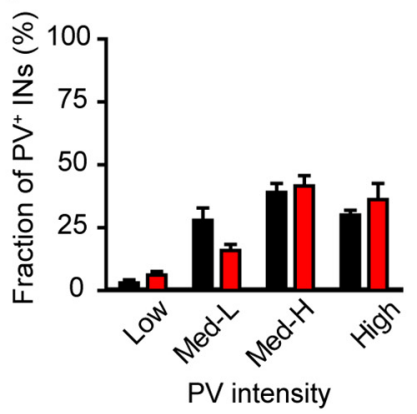

Figure 5. Atypical high-PV-network configuration in the M1 cortex of Mecp2 KO mice. $\boldsymbol{A}$, Representative images showing PV expression in layer II/III of M1 cortex in both WT and Mecp2 KO mice at P56. Histograms showing quantitative analysis of PV ${ }^{+}$cell density at P56 (B), PV mean fluorescence intensity $(\boldsymbol{C})$, cumulative $(\boldsymbol{D})$ and binned $(\boldsymbol{E})$ frequency distribution of PV cells intensity in WT and Mecp2 KO mice. $\boldsymbol{F}$, Representative images showing PV expression in layer II/III of M1 cortex in both WT and Mecp2 KO mice at P28. Histograms showing quantitative analysis of PV cell density $(\boldsymbol{G})$, PV mean fluorescence intensity $(\boldsymbol{H})$, cumulative $(\boldsymbol{I})$ and binned $(\boldsymbol{J})$ frequency distribution of PV cells intensity in WT and Mecp2 KO mice at P28. $n=6$ mice per genotype at P56 and $n=4$ mice per genotype at P28. Student's $t$ test: $* p<$ $0.05, * * p<0.01$, Mann-Whitney $U$ test for $\boldsymbol{D}, \boldsymbol{I}:{ }^{\#} p<0.05$; ${ }^{\# \#} p<0.01$. Scale bars $=100 \mu \mathrm{m}$.

number of cortical INs expressing higher levels of PV.

It has been previously proposed by Donato et al. (2013) that transitions among different levels of PV expression in $\mathrm{M} 1$ represent a molecular signature of $\mathrm{PV}^{+}$IN plasticity reflecting motor learning. To evaluate both motor performance and learning-dependent plasticity of $\mathrm{PV}^{+}$INs in Mecp2 KO mice, we trained P56 mice on the accelerating rotarod task (RR) for 2 days (8 trials) while activity control (AC) mice ran at a constant speed across trials. Although rotarod performance improved in consecutive trials in both genotypes, P56 Mecp2 KO mice spent significantly less time on the rod than WT animals (Fig. 6A) as previously reported (Stearns et al., 2007). Interestingly, the intensity of PV expression shown by $A C$ mice was only subtly different between genotypes in M1 cortex (Fig. 6B) as indicated by cumulative frequency distribution analysis (Fig. 6C), probably because AC test may have modified
PV expression in $\mathrm{M} 1$ reducing the differences between genotypes. Intriguingly, while WT-RR mice exhibited a modest leftward shift toward lower PV intensity level, $\mathrm{PV}^{+}$ INs in Mecp2 KO-RR mice showed a robust rightward shift response (Fig. 6C). Moreover, subclasses analysis indicated that Mecp2 KO-RR mice show a reduced percentage of medium-high-PV cells compared to Mecp2 $\mathrm{KO}-\mathrm{AC}$ and a robust increase of the high-PV fraction compared to both Mecp2 KO-AC and, importantly, WT-RR mice (Fig. 6D). Finally, two-way ANOVA analysis revealed a significant effect of both genotype and $R R$ training specifically for the high-PV subclass (Fig. 6D).

Remarkably, we found that a greater fraction of high$\mathrm{PV}^{+}$INs negatively correlated with the motor performance of RR-trained animals (Fig. 6E), suggesting that higher PV expression adversely affects behavioral learning. These data indicate that molecular plasticity of $\mathrm{PV}^{+}$INs induced by motor learning is atypical in behaviorally impaired 
A

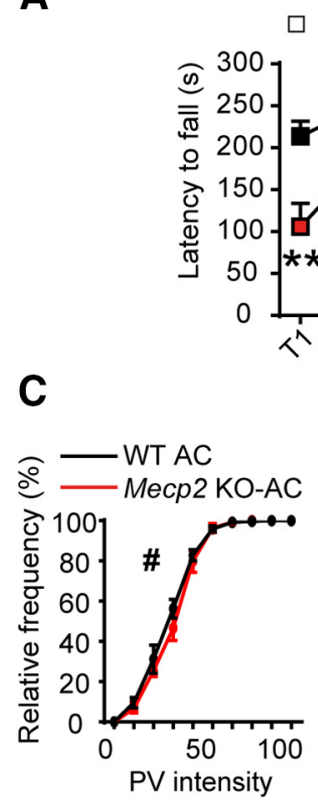

$\square$ WT-RR $\square$ Mecp2 KO-RR

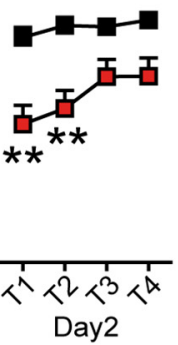

B
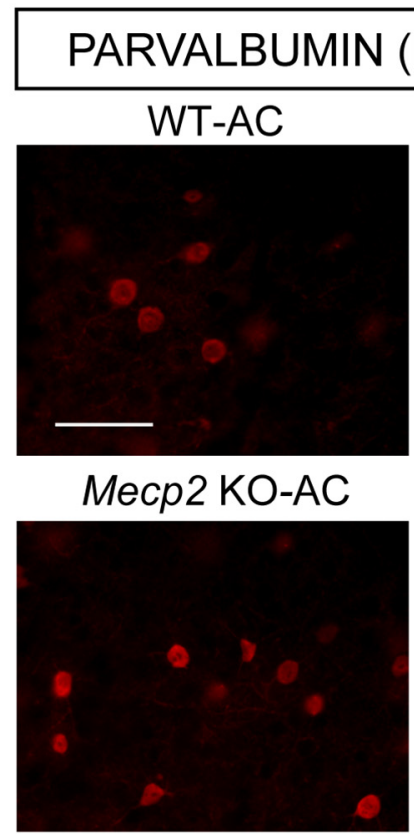

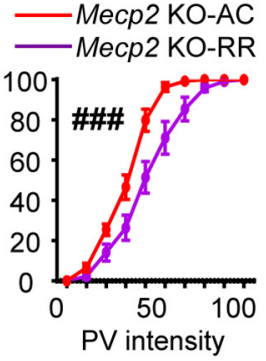

$\mathbf{E}$
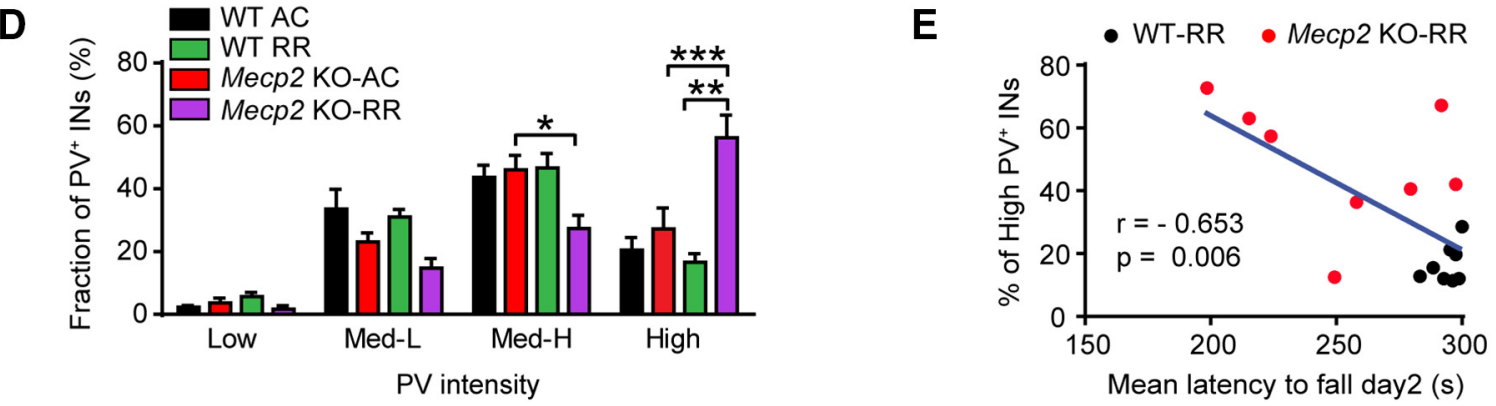

Figure 6. Motor learning-induced plasticity of PV network is impaired in symptomatic Mecp2 KO mice. A, Latency to fall (seconds) from an accelerating rotating rod in P56 Mecp2 KO mice and WT littermates. Graphs show data of first and last trials/d (T1-4), for two consecutive days (day 1-2). B, Representative images of PV immunofluorescence in layer II/III INs of the M1 cortex in both WT and Mecp2 KO P56 mice after AC or RR tasks. Cumulative $(\boldsymbol{C})$ and binned $(\boldsymbol{D})$ frequency distribution of PV cells intensity in layer II/III of M1 cortex, in Mecp2 KO mice and WT littermates after AC or RR tasks. (E) Correlation analysis between the mean latency to fall (seconds) from the rod on day 2 and the fraction of high $\mathrm{PV}^{+}$INs in Mecp2 KO mice and WT littermates. $n=6 \mathrm{WT}-\mathrm{AC}$ mice, 5 Mecp2 KO-AC mice, 6 WT-RR mice, and 5 Mecp2 KO-RR mice for $\boldsymbol{C}$ and $\boldsymbol{D}$. $n=9$ WT-RR mice and 9 Mecp2 KO-RR mice for $\boldsymbol{A}$ and $\boldsymbol{E}$. Two-way ANOVA and Bonferroni posthoc tests for $\boldsymbol{A}$ and $\boldsymbol{D}$ : $* p<0.05, * * p<0.01, * * * p<0.001$; Mann-Whitney $U$ test for $\boldsymbol{C}$ : \# $p$ $<0.05,{ }^{\# \#} p<0.01,{ }^{\# \# \#} p<0.001$; Pearson's $r$ for $\boldsymbol{E}$. Scale bar $=100 \mu \mathrm{m}$.

Mecp2 KO mice, resulting in an abnormal increase of cells expressing high PV levels in the M1 cortex.

\section{Atypical structural synaptic plasticity on $\mathrm{PV}^{+}$INs in behaviorally impaired Mecp2 KO mice}

Changes in PV networks induced by behavioral experience rely on the structural plasticity of both excitatory and inhibitory synapses onto $\mathrm{PV}^{+}$INs (Donato et al., 2013). Therefore, we investigated whether the atypical shift of the distribution of PV-cell subclasses occurring in Mecp2 KO mice could be produced by aberrant changes of $\mathrm{E} / \mathrm{l}$ connectivity reaching $\mathrm{PV}^{+}$INs. The density of both excitatory $\left(\mathrm{VGLUT1}^{+}\right)$and inhibitory $\left(\mathrm{VGAT}^{+}\right)$presynaptic terminals onto dendrites and somata of layer II/III PV ${ }^{+}$INs in $\mathrm{M} 1$ cortex was quantitatively evaluated on confocal images (Fig. 7A). Similar to $\mathrm{S} 1$ cortex of untrained animals, the M1 cortex of Mecp2 KO-AC mice showed a higher density of excitatory boutons decorating both dendritic and soma compartments of $\mathrm{PV}^{+}$INs compared with WT animals (Fig. $7 B, C$ ). Intriguingly, the density of excitatory boutons contacting PV-labeled dendrites was increased in Mecp2 KO-RR mice (Fig. 7B) while there were no changes in WT-RR animals. When we extended this analysis to the somata of $\mathrm{PV}^{+}$cells, we found that RR task produced a significant reduction of VGLUT1 ${ }^{+}$puncta that was similar between genotypes (Fig. 7C). In sum, following RR training, Mecp2 KO mice showed a higher density of putative excitatory synapses contacting $\mathrm{PV}^{+} \mathrm{INs}$ than WT animals (Fig. 7B, C).

When we analyzed the density of $\mathrm{VGAT}^{+}$puncta onto $\mathrm{PV}^{+}$INs, we found that there were no differences between genotypes in AC condition (Fig. 7D, E). Interestingly, we found that the density of $\mathrm{VGAT}^{+}$puncta onto $\mathrm{PV}^{+}$dendrites was increased in Mecp2 KO-RR mice (Fig. 7D) but 

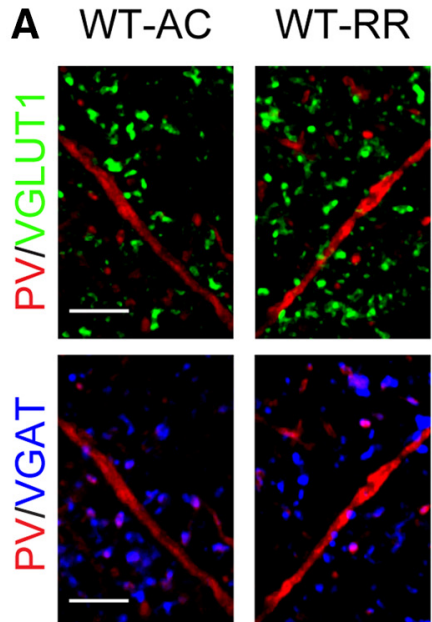

Mecp2 KO Mecp2 KO

$A C$
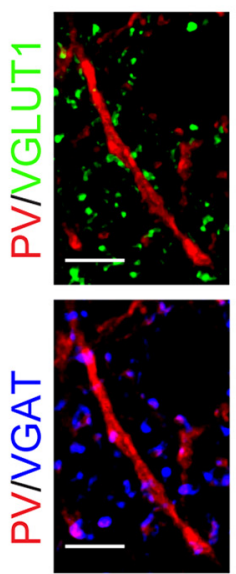

B
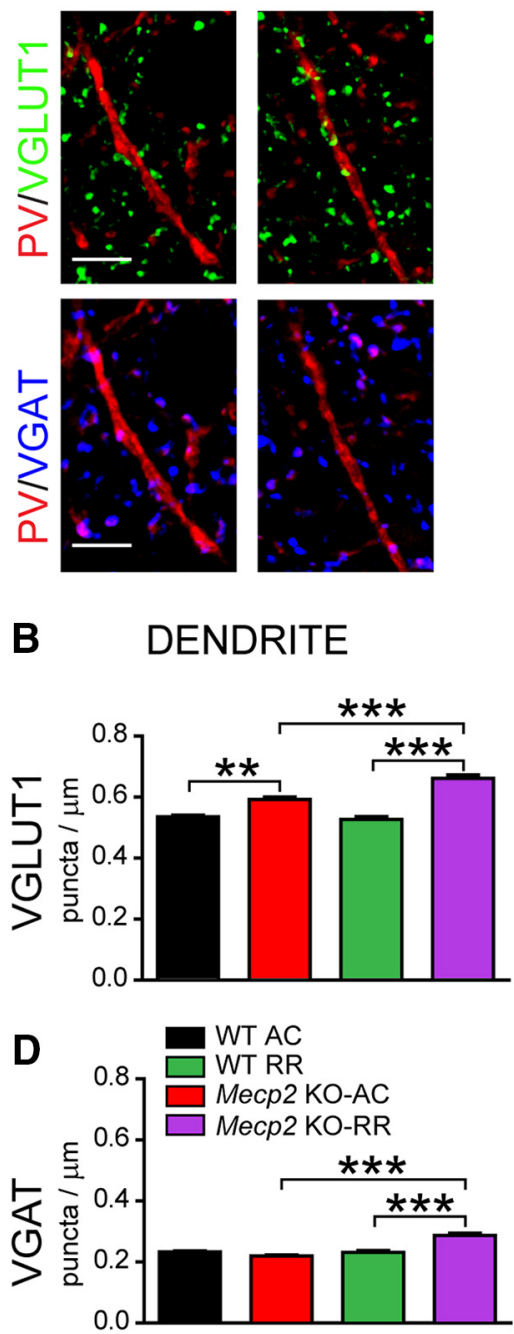

DENDRITE
WT-AC
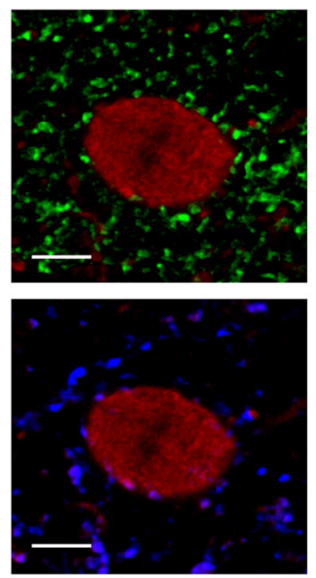

Mecp2 KO

$A C$
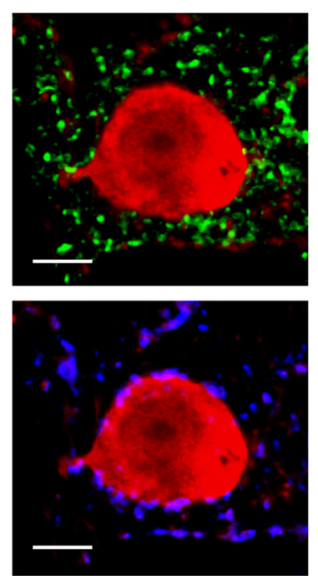

C

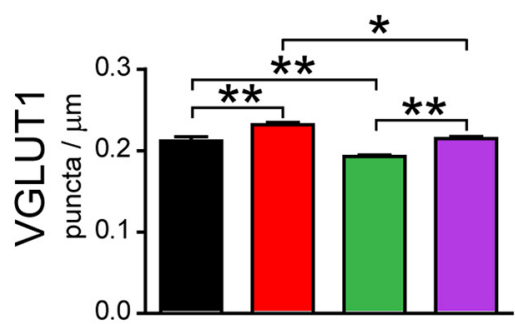

E

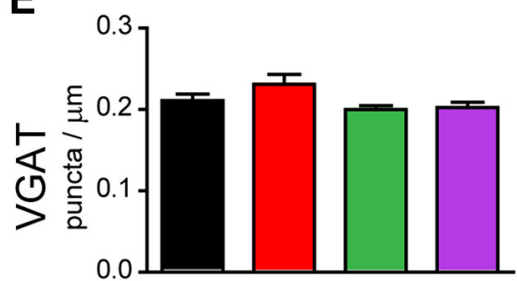

Figure 7. Motor learning produces atypical structural synaptic plasticity of inputs converging on $\mathrm{PV}^{+} \mathrm{INs}$ in Mecp2 KO mice. A, Representative confocal images of excitatory VGLUT1 ${ }^{+}$(green) and inhibitory VGAT ${ }^{+}$(blue) puncta apposed to $\mathrm{PV}^{+}$(red) dendrites and somata in layer II/III of M1 cortex in AC- and RR-trained WT and Mecp2 KO mice. B, C, Histograms showing quantitative analysis of VGLUT1 ${ }^{+}$puncta density on dendrites $(\boldsymbol{B})$ and somata $(\boldsymbol{C})$ of $\mathrm{PV}^{+} \mathrm{INs}$ after AC and RR training. $\boldsymbol{D}, \boldsymbol{E}$, Histograms showing quantitative analysis of VGAT ${ }^{+}$puncta density on dendrites $(\boldsymbol{D})$ and somata $(\boldsymbol{E})$ of $\mathrm{PV}^{+}$INs after AC and RR training. Dendrites: $n=5 \mathrm{WT}$ and 5 Mecp2 KO mice; somata: $n=6 \mathrm{WT}$ and $5 \mathrm{Mecp} 2$ KO mice. Two-way ANOVA and Bonferroni posthoc tests: $* p<0.05, * * p<0.01, * * * p<0.001$. Scale bar $=5 \mu \mathrm{m}$. 
not in WT-RR animals, resulting in a significant difference in the number of $\mathrm{VGAT}^{+}$terminals between genotypes (Fig. 7D). Finally, our analysis showed that RR did not change the number of $\mathrm{VGAT}^{+}$puncta along the somata of $\mathrm{PV}^{+}$INs in both genotypes (Fig. 7E). Altogether, these data indicate that behaviorally impaired Mecp2 KO mice show an atypical activity-dependent structural synaptic plasticity on $\mathrm{PV}^{+}$INs in the M1 cortex that likely produces an aberrant increase of high-PV cell density after motor learning.

\section{Atypical PV levels in the M1 cortex correlate with both symptom progression and motor coordination disabilities in female Mecp2 heterozygous mice}

Finally, we assessed $\mathrm{PV}$ expression and $\mathrm{PV}^{+}$cell plasticity in female Mecp2 Het mice, which exhibit mosaic expression of Mecp2 that resembles the human condition (Katz et al., 2012). As in RTT individuals, female Mecp2 Het mice have an apparently typical early development, followed by the emergence of several RTT-like symptoms, such as motor impairments, that worsen during the progression of the disease. We analyzed asymptomatic (2-mo-old), presymptomatic (4-mo-old), and severely symptomatic (8-mo-old) mice (De Filippis et al., 2010) to evaluate whether changes in the configuration of $\mathrm{PV}$ networks in $\mathrm{M} 1$ cortex correlate with symptom appearance (Fig. $8 A, D, G$ ). The cumulative frequency distribution analysis revealed a very subtle, although significant, increase of PV intensity in symptomatic Mecp2 Het mice compared with WT mice, while younger mutants were unaffected (Fig. 8B, E, H). On the other hand, while the analysis of the percentage of PV-cell subclasses showed no differences between genotypes at 2 mo of age (Fig. $8 C$ ), we found an increase in the high-PV fraction in presymptomatic Mecp2 Het mice compared with WT animals (Fig. 8F), a change that was maintained in severely symptomatic Mecp2 Het mice (Fig. 8).

We next tested motor coordination in female Mecp2 Het mice at $8 \mathrm{mo}$ of age and found that these animals showed a significant impairment compared with WT controls in the rotarod task (Fig. 8J). Intriguingly, as for Mecp2 KO mice, a higher fraction of the high-PV cell INs subclass negatively correlated with motor performance of RR-female animals (Fig. 8K). Taken together, our analysis indicates that the PV network is affected in Mecp2 Het female mice and that atypically elevated PV expression is associated with worsened motor coordination shown by these mice.

\section{Discussion}

Dysfunctions of synaptic connectivity and plasticity are thought to be important cellular determinants of RTT and other disorders associated with sensory-motor and intellectual disabilities (Boggio et al., 2010; Zoghbi and Bear, 2012). Revealing the nature of these deficits and identifying the impacted brain areas are crucial to guide the progression and targeting of efficient therapies for these conditions (Shepherd and Katz, 2011; Cortés-Mendoza et al., 2013; $\mathrm{Na}$ et al., 2013). Moreover, it is of great importance to characterize the developmental timeline of specific pathologic alterations to establish a putative therapeutic window. In this study, we investigated the putative molecular, synaptic and network determinants underlying motor control and somatosensory processing in Mecp2 mutant mice. Our results indicate that MeCP2 expression is required for correct synaptic remodeling that regulates the degree of $\mathrm{PV}^{+} \mathrm{IN}$ network plasticity in response to sensory-motor behavioral learning.

We found for the first time that symptomatic Mecp2 KO mice show a robust shift of $\mathrm{PV}^{+} \mathrm{IN}$ networks in both $\mathrm{S} 1$ and $\mathrm{M} 1$ cortices toward high-PV expression configuration, a condition reflecting atypical plasticity of these INs (Donato et al., 2013). This high-PV configuration was associated with a shift of the E/I input balance toward increased excitatory connectivity that is already present in presymptomatic mutant mice showing only subtle changes of PV expression. When we tested the functional implications of these changes in Mecp2 KO mice, we found a significant early-onset reduction of stimulusinduced network activity in acute cortical slices consistent with hyperactivated PV inhibitory circuits. Finally, we hypothesized that such altered state of activity and connectivity could impair synaptic plasticity in cortical circuits relevant for motor learning. In support of this hypothesis, we found that lack of MeCP2 disrupts activity-induced pattern of both $\mathrm{PV}$-expression and $\mathrm{E} / \mathrm{I}$ input rearrangements in $\mathrm{PV}^{+} \mathrm{INs}$ of the $\mathrm{M} 1$ cortex, processes that are associated with the encoding of new motor experiences in this area (Donato et al., 2013). That atypical PV-level adjustment is likely to underlie motor impairments in RTT is strongly supported by our results showing that the larger number of high-PV ${ }^{+}$INs that we found in M1 cortex of both male Mecp2 KO and female Mecp2 Het mice negatively correlates with behavioral performance.

Our study establishes that in Mecp2 KO mice there is an increase of PV expression in both S1 and M1 cortices, similar to what was previously reported in other neocortical areas (Durand et al., 2012; Patrizi et al., 2015; Krishnan et al., 2015, 2017), further highlighting the role of $\mathrm{PV}$ regulation in the progression of RTT pathology. Here, we show for the first time that in layer II/III of both S1 and M1 cortices there is an increase in the density of excitatory presynaptic puncta onto dendrites of $\mathrm{PV}^{+} \mathrm{INs}$ in P28 and P56 Mecp2 KO mice compared with WT littermates. Thus, our data indicate that Mecp2 loss enhances excitatory synaptogenesis on $\mathrm{PV}^{+}$cells, which begins during the period of apparent typical development preceding overt pathologic symptoms, that likely results in higher PV expression. Interestingly, in symptomatic Mecp2 KO mice there is a further aberrant remodeling of putative excitatory synapses occurring at the somata of $\mathrm{PV}^{+}$cells after synaptogenesis is completed that may represent a primary cause of the aggravation of RTT symptoms. Moreover, our data indicate that the increased density of excitatory terminals onto $\mathrm{PV}^{+} \mathrm{INs}$ is not determined as a secondary consequence of the smaller cell body area shown by mutant mice (see Tomassy et al., 2014), but instead reflects aberrant excitatory synapse formation and/or maintenance on PV interneurons lacking MeCP2.

In line with this idea, we found that the amplitude and spatio-temporal spread of neuronal depolarizations evoked in layer II/III were significantly smaller in both presymptomatic and symptomatic Mecp2 KO mice. 

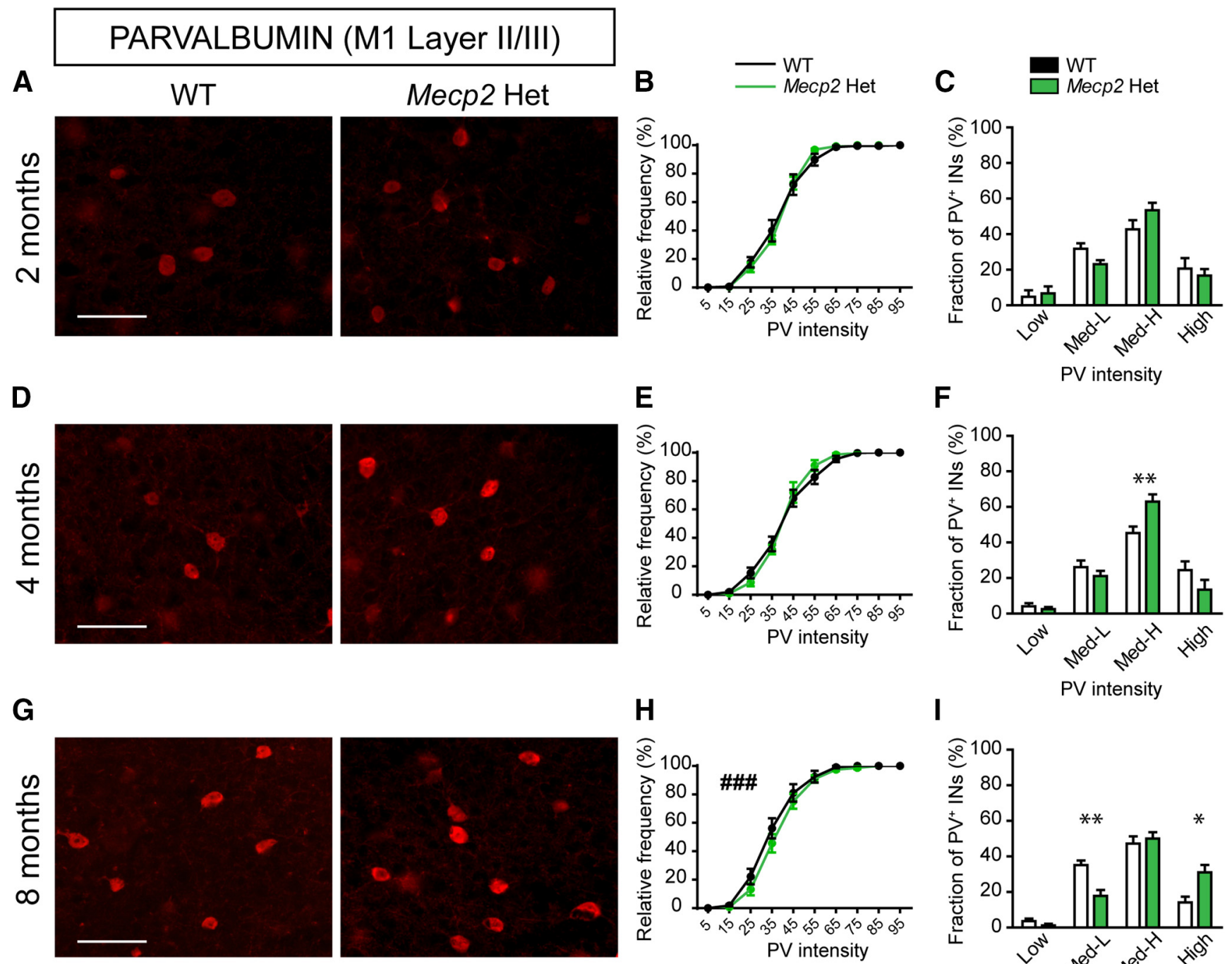

$\mathbf{F}$

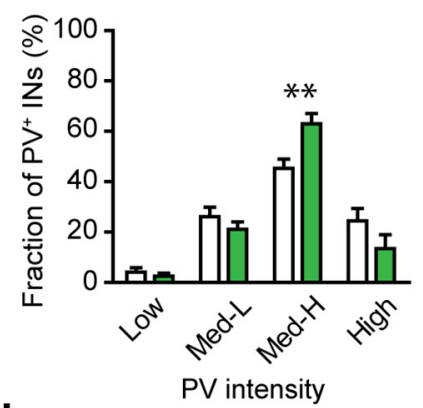

$\mathbf{J}$

K

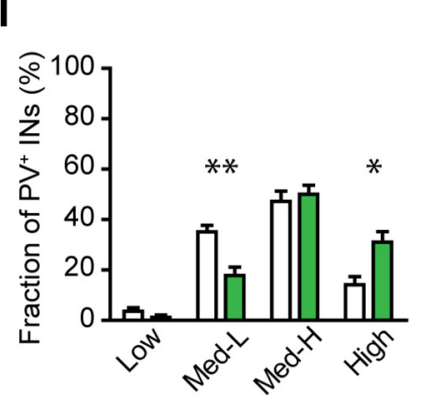

PV intensity
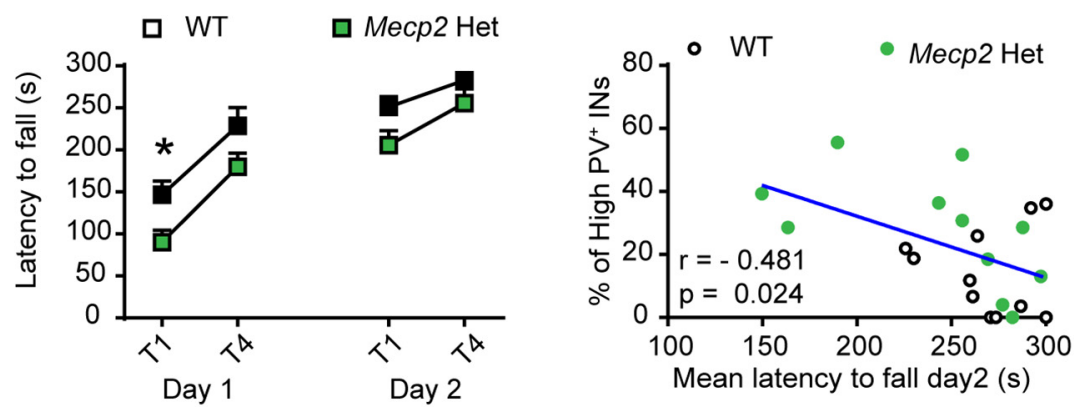

Figure 8. Atypical high-PV expression in the M1 cortex of female Mecp2 Het mice correlates with motor impairments. Representative images showing PV expression in layer II/III of M1 cortex in both WT and Mecp2 Het mice at $2(\boldsymbol{A}), 4(\boldsymbol{D})$, and $8(\boldsymbol{G})$ months of age. Cumulative $(\boldsymbol{B}, \boldsymbol{E}, \boldsymbol{H})$ and binned $(\boldsymbol{C}, \boldsymbol{F}, \boldsymbol{I})$ frequency distribution of PV cells intensity in WT and Mecp2 Het M1 cortex at $2(\boldsymbol{B}, \boldsymbol{C}), 4$ $(\boldsymbol{E}, \boldsymbol{F})$, and $8(\boldsymbol{H}, \boldsymbol{I})$ months of age. $\boldsymbol{J}$, Latency to fall (seconds) from an accelerating rotating rod in 8-mo-old Mecp2 Het mice and WT littermates. Graphs show data of first and last trials/d (T1-4), for two consecutive days (day 1-2). $\boldsymbol{K}$, Correlation analysis between the mean latency to fall (seconds) from the rod on day 2 and the fraction of high PV ${ }^{+}$INs in 8-mo-old Mecp2 Het and WT females. A-I: $n=6$ WT and 6 Mecp2 Het mice; $\boldsymbol{J}, \boldsymbol{K}: n=11$ WT and 11 Mecp2 Het mice. Mann-Whitney $U$ test for $\boldsymbol{B}, \boldsymbol{E}, \boldsymbol{H}:{ }^{\# \# \#} p<0.001 ;$ Student's $t$ test for $\boldsymbol{C}, \boldsymbol{F}, \boldsymbol{I}: * p<0.05, * * p<0.01$; two-way ANOVA and Bonferroni posthoc tests for $\boldsymbol{J}$ : $* p<0.05$, $* * p<0.01$; Pearson's $r$ for E. Scale bar $=100 \mu \mathrm{m}$. 
Because our data suggest that supernumerary $\mathrm{PV}^{+} \mathrm{INs}$ (see also Tomassy et al., 2014) in layer II/III of Mecp2 KO mice are hyperactive, it is feasible to suppose that excessive inhibitory drive tampers with both network responses and somatosensory stimuli processing in S1. Although our current results are consistent with observations in V1 cortex of Mecp2 KO mice (Durand et al., 2012), in this previous study the reduction of VSD propagation response was recognized as stronger $\mathrm{PV}^{+}$cells-to-layer IV pyramidal neuron connectivity. As an alternative, we here suggest that, as a result of excessive excitatory inputs, lack of Mecp2 leads to atypical hyperactivation of $\mathrm{PV}^{+}$ Ins, resulting in decreased cortical network activity. Because these two mechanisms are not mutually exclusive, and since it has been recently proposed that the effectiveness of GABA as an inhibitory transmitter may be altered in Mecp2 KO mice (Banerjee et al., 2016), it will be necessary to further extend these studies to dissect out the mechanisms leading to atypical cortical activity in the absence of Mecp2.

Based on our findings, we propose a dual role of MeCP2 in regulating $\mathrm{PV}^{+}$IN connectivity in both $\mathrm{S} 1$ and M1: early, during the developmental processes that shape neural circuits; and late, when synaptic remodeling is mainly produced by experience-dependent plasticity. The early synaptic events caused by Mecp2 loss primarily stem from defects involving the $\mathrm{PV}^{+}$IN subpopulation, because synaptic connectivity on $\mathrm{CR}^{+}$ neurons in presymptomatic Mecp2 $\mathrm{KO}$ mice was like that of WT animals. Moreover, our results indicate that the worsening of the pathologic symptoms observed in developing Mecp2 KO mice are coincidental with the progressive engagement of additional subclasses of INs, leading to a more extensive pathology of cortical circuits, resulting in altered network activity levels, and hindering the ability of those networks to undergo experience-dependent plasticity. Because it has been previously shown that in the neocortex $\mathrm{CR}^{+}$cells mostly target $\mathrm{PV}^{+}$and somatostatin-expressing cells (Gonchar and Burkhalter, 1999), we cannot exclude that an enhanced excitatory input on $\mathrm{CR}^{+}$cells may represent a homeostatic mechanism to compensate for $\mathrm{PV}^{+}$ IN hyperfunctionality. Previous studies have shown that inhibitory synapses from different subclasses of INs target $\mathrm{PV}^{+}$cells on distinct subcellular compartments: $\mathrm{PV}^{+}$puncta, and less frequently somatostatin ${ }^{+}$terminals, are found to preferentially target the dendrites, while somatic inhibitory inputs targeting $\mathrm{PV}^{+}$INs mainly originate from cells expressing vasoactive intestinal peptide (Hioki et al., 2013). Therefore, consistent with previous observation in V1 (Krishnan et al., 2015), our current findings show that inter-PV ${ }^{+}$cell communication is selectively affected in the S1 cortex of Mecp2 KO mice starting at P28.

In line with a late role of Mecp2, we find that, unlike WT mice, PV networks in M1 cortex of symptomatic Mecp2 $\mathrm{KO}$ mice shifts toward high-PV expression when performing a rotarod learning task. Consistently, we observed an atypical increase of excitatory connectivity on $\mathrm{PV}^{+}$INs in Mecp2 KO mice after rotarod learning, accompanied by a weak increase of inhibitory inputs. Our study indicates for the first time that $\mathrm{PV}^{+}$cells in Mecp2 $\mathrm{KO}$ mice undergo aberrant molecular and synaptic changes during the performance and learning of a motor task. Activitydependent fluctuations of PV expression in M1 cortex of WT mice have been previously associated with synaptic remodeling converging onto these cells (Donato et al., 2013). It is important to note that we have not been able to fully recapitulate the effect size on both PV expression and synaptic remodeling in M1 cortex of WT animals after $\mathrm{RR}$, although we report changes that are coherent with this previous study. These discrepancies are likely due to differences in the anatomic approaches, the criteria used to subdivide $\mathrm{PV}^{+} \mathrm{IN}$ classes, and importantly, mouse strains. Finally, our study is the first to demonstrate that motor learning-induced synaptic plasticity occurs both on dendritic and somatic compartments of $\mathrm{PV}^{+} \mathrm{INs}$, and that lack of Mecp2 disrupts structural changes of excitatory connectivity onto these cells. Interestingly, it is known that MeCP2 mutation affects the expression of Npas4, a transcription factor that regulates the E/I balance onto INs by modulating the density of excitatory puncta (Ebert et al., 2013). Thus, additional experiments will clarify the molecular mechanisms responsible for the activity-dependent remodeling of synaptic inputs onto $\mathrm{PV}^{+} \mathrm{INs}$, both in typically developing mice and in Mecp2 models.

Finally, we found a similar PV-expression phenotype also in M1 cortex of Mecp2 Het female mice, suggesting that physiologic levels of expression of MeCP2 protein are important for the configuration and plasticity of PV networks and that a partial reduction of MeCP2 expression is sufficient to interfere with this process. Our study indicates that the increment of PV levels occurs when female Mecp2 Het mice start to show clear symptoms (Samaco et al., 2013), after a period (i.e., between 2 and 4 months) of typical PV-network configuration. Importantly, because we found a negative correlation between the fraction of high-PV cells in M1 cortex and the motor performance in Mecp2 Het female mice, it is tempting to speculate that a partial inhibition of $\mathrm{PV}^{+}$INs would be beneficial for the motor impairments in RTT.

\section{References}

Amir RE, Van den Veyver IB, Wan M, Tran CQ, Francke U, Zoghbi HY (1999) Rett syndrome is caused by mutations in X-linked MECP2, encoding methyl-CpG-binding protein 2. Nat Genet 23:185-188. CrossRef Medline

Armstrong DD, Dunn K, Antalffy B (1998) Decreased dendritic branching in frontal, motor and limbic cortex in Rett syndrome compared with trisomy 21. J Neuropathol Exp Neurol 57:10131017. Medline

Banerjee A, Rikhye RV, Breton-Provencher V, Tang X, Li C, Li K, Runyan CA, Fu Z, Jaenisch R, Sur M (2016) Jointly reduced inhibition and excitation underlies circuit-wide changes in cortical processing in Rett syndrome. Proc Natl Acad Sci USA 15:E7287E7296. CrossRef

Boggio EM, Lonetti G, Pizzorusso T, Giustetto M (2010) Synaptic determinants of rett syndrome. Front Synaptic Neurosci 2:28. CrossRef Medline

Belichenko PV, Wright EE, Belichenko NP, Masliah E, Li HH, Mobley WC, Francke U (2009) Widespread changes in dendritic and ax- 
onal morphology in Mecp2-mutant mouse models of Rett syndrome: evidence for disruption of neuronal networks. J Comp Neur 20:240-258. CrossRef

Cardin JA, Carlén M, Meletis K, Knoblich U, Zhang F, Deisseroth K, Tsai LH, Moore Cl (2009) Driving fast-spiking cells induces gamma rhythm and controls sensory responses. Nature 459:663-667. CrossRef Medline

Caroni P (2015) Regulation of Parvalbumin Basket cell plasticity in rule learning. Biochem Biophys Res Commun 24:100-103. CrossRef

Chao HT, Chen H, Samaco RC, Xue M, Chahrour M, Yoo J, Neul JL, Gong S, Lu HC, Heintz N, Ekker M, Rubenstein JL, Noebels JL, Rosenmund C, Zoghbi HY (2010) Dysfunction in GABA signalling mediates autism-like stereotypies and Rett syndrome phenotypes. Nature 468:263-269. CrossRef Medline

Chen RZ, Akbarian S, Tudor M, Jaenisch R (2001) Deficiency of methyl-CpG binding protein-2 in CNS neurons results in a Rett-like phenotype in mice. Nat Genet 27:327-331. CrossRef Medline

Cortés-Mendoza J, Díaz de León-Guerrero S, Pedraza-Alva G, Pérez-Martínez $L$ (2013) Shaping synaptic plasticity: the role of activity-mediated epigenetic regulation on gene transcription. Int $\mathrm{J}$ Dev Neurosci 31:359-369. CrossRef Medline

De Filippis B, Ricceri L, Laviola G (2010) Investigating Rett syndrome through genetic mouse models: presymptomatic, clearly symptomatic phases, and innovative therapeutic approaches. In Kalueff A. and Bergner C (ed.), Transgenic and Mutant Tools to Model Brain Disorders, Neuromethods, Vol.44 (pp. 151-178). Totowa, NJ: Humana Press.

Donato F, Rompani SB, Caroni P (2013) Parvalbumin-expressing basket-cell network plasticity induced by experience regulates adult learning. Nature 504:272-276. CrossRef Medline

Donato F, Chowdhury A, Lahr M, Caroni P (2015) Early- and late-born parvalbumin basket cell subpopulations exhibiting distinct regulation and roles in learning. Neuron 85:770-786. CrossRef Medline

Downs J, Stahlhut M, Wong K, Syhler B, Bisgaard AM, Jacoby $\mathrm{P}$, Leonard H (2016) Validating the Rett syndrome gross motor scale. PLoS One 11:e0147555. CrossRef Medline

Durand S, Patrizi A, Quast KB, Hachigian L, Pavlyuk R, Saxena A, Carninci P, Hensch TK, Fagiolini M (2012) NMDA receptor regulation prevents regression of visualcortical function in the absence of Mecp2. Neuron 20:1078-1090. CrossRef

Ebert DH, Gabel HW, Robinson ND, Kastan NR, Hu LS, Cohen S, Navarro AJ, Lyst MJ, Ekiert R, Bird AP, Greenberg ME (2013) Activity-dependent phosphorylation of MeCP2 threonine 308 regulates interaction with NCoR. Nature 18:341-345. CrossRef

Fukuda T, Itoh M, Ichikawa T, Washiyama K, Goto Y (2005) Delayed maturation of neuronal architecture and synaptogenesis in cerebral cortex of Mecp2-deficient mice. J Neuropathol Exp Neurol 64:537-544. Medline

Franklin KBJ, Paxinos G (1997) The Mouse Brain in Stereotaxic Coordinates. San Diego, CA: Academic Press.

Guy J, Hendrich B, Holmes M, Martin JE, Bird A (2001) A mouse Mecp2-null mutation causes neurological symptoms that mimic Rett syndrome. Nat Genet 27:322-326. CrossRef Medline

Gonchar Y, Burkhalter A (1999) Connectivity of GABAergic calretininimmunoreactive neurons in rat primary visual cortex. Cereb Cortex 9:683-696. Medline

He LJ, Liu N, Cheng TL, Chen XJ, Li YD, Shu YS, Qiu ZL, Zhang XH (2014) Conditional deletion of Mecp2 in parvalbumin-expressing GABAergic cells results in the absence of critical period plasticity. Nat Commun 5:5036. CrossRef Medline

Hioki H, Okamoto S, Konno M, Kameda H, Sohn J, Kuramoto E, Fujiyama F, Kaneko T (2013) Cell type-specific inhibitory inputs to dendritic and somatic compartments of parvalbumin-expressing neocortical interneuron. J Neurosci 33:544-555. CrossRef Medline

Ito-Ishida A, Ure K, Chen H, Swann JW, Zoghbi HY (2015) Loss of MeCP2 in parvalbumin-and somatostatin-expressing neurons in mice leads to distinct Rett syndrome-like phenotypes. Neuron 88:651-658. CrossRef Medline

Isomura Y, Harukuni R, Takekawa T, Aizawa H, Fukai T (2009) Microcircuitry coordination of cortical motor information in self- initiation of voluntary movements. Nat Neurosci 12:1586-1593. CrossRef Medline

Katz DM, Berger-Sweeney JE, Eubanks JH, Justice MJ, Neul JL, Pozzo-Miller L, Blue ME, Christian D, Crawley JN, Giustetto M, Guy J, Howell CJ, Kron M, Nelson SB, Samaco RC, Schaevitz LR, St HillaireClarke C, Young JL, Zoghbi HY, Mamounas LA (2012) Preclinical research in Rett syndrome: setting the foundation for translational success. Dis Model Mech 5:733-745. CrossRef Medline

Krishnan K, Lau BY, Ewall G, Huang ZJ, Shea SD (2017) MECP2 regulates cortical plasticity underlying a learned behaviour in adult female mice. Nat Commun 8:14077. CrossRef Medline

Lyst MJ, Bird A (2015) Rett syndrome: a complex disorder with simple roots. Nat Rev Genet 16:261-275. CrossRef Medline

Krishnan K, Wang BS, Lu J, Wang L, Maffei A, Cang J, Huang ZJ (2015) MeCP2 regulates the timing of critical period plasticity that shapes functional connectivity in primary visual cortex. Proc Natl Acad Sci USA 112:E4782-E4791. CrossRef Medline

Na ES, Nelson ED, Kavalali ET, Monteggia LM (2013) The impact of MeCP2 loss- or gain-of-function on synaptic plasticity. Neuropsychopharmacology 38:212-219. CrossRef Medline

Neul JL, Kaufmann WE, Glaze DG, Christodoulou J, Clarke AJ, Bahi-Buisson N, Leonard H, Bailey ME, Schanen NC, Zappella M, Renieri A, Huppke P, Percy AK (2010) Rett syndrome: revised diagnostic criteria and nomenclature. Ann Neurol 68:944-950. CrossRef Medline

Patrizi A, Picard N, Simon AJ, Gunner G, Centofante E, Andrews NA, Fagiolini M (2015) Chronic administration of the N-methyl-Daspartate receptor antagonist ketamine improves Rett syndrome phenotype. Biol Psychiatry 79:755-764. CrossRef Medline

Pi HJ, Hangya B, Kvitsiani D, Sanders JI, Huang ZJ, Kepecs A (2013) Cortical interneurons that specialize in disinhibitory control. Nature 503:521-524. CrossRef Medline

Pizzo R, Gurgone A, Castroflorio E, Amendola E, Gross C, SassoèPognetto M, Giustetto M (2016) Lack of Cdkl5 disrupts the organization of excitatory and inhibitory synapses and parvalbumin interneurons in the primary visual cortex. Front Cell Neurosci 10:261. CrossRef Medline

Sachidhanandam S, Sermet BS, Petersen CC (2016) Parvalbuminexpressing GABAergic neurons in mouse barrel cortex contribute to gating a goal-directed sensorimotor transformation. Cell Rep 15:700-706. CrossRef

Samaco RC, McGraw CM, Ward CS, Sun Y, Neul JL, Zoghbi HY (2013) Female Mecp2( $\left(^{+} /-\right)$mice display robust behavioral deficits on two different genetic backgrounds providing a framework for pre-clinical studies. Hum Mol Genet 22:96-109. CrossRef Medline

Shepherd GM, Katz DM (2011) Synaptic microcircuit dysfunction in genetic models of neurodevelopmental disorders: focus on Mecp2 and Met. Curr Opin Neurobiol 21:827-833. CrossRef Medline

Smeets EE, Pelc K, Dan B (2012) Rett syndrome. Mol Syndromol 2:113-127. Medline Medline

Sohal S, Zhang F, Yizhar O, Deisseroth K (2009) Parvalbumin neurons and gamma rhythms enhance cortical circuit performance. Nature 459:698-702. CrossRef

Stearns NA, Schaevitz LR, Bowling H, Nag N, Berger UV, BergerSweeney J (2007) Behavioral and anatomical abnormalities in Mecp2 mutant mice: a model for Rett syndrome. Neuroscience 146:907-921. CrossRef

Temudo T, Ramos E, Dias K, Barbot C, Vieira JP, Moreira A, Calado E, Carrilho I, Oliveira G, Levy A, Fonseca M, Cabral A, Cabral P, Monteiro JP, Borges L, Gomes R, Santos M, Sequeiros J, Maciel $P$ (2008) Movement disorders in Rett syndrome: an analysis of 60 patients with detected MECP2 mutation and correlation with mutation type. Mov Disord 23:1384-1390. CrossRef Medline

Tomassy GS, Morello N, Calcagno E, Giustetto M (2014) Developmental abnormalities of cortical interneurons precede symptoms onset in a mouse model of Rett syndrome. J Neurochem 131:115127. CrossRef 
Volman V, Behrens MM, Sejnowski TJ (2011) Downregulation of parvalbumin at cortical GABA synapses reduces network gamma oscillatory activity. J Neurosci 31:18137-18148. CrossRef Medline

Zhang W, Peterson M, Beyer B, Frankel WN, Zhang ZW (2014) Loss of MeCP2 from forebrain excitatory neurons leads to cortical hyperexcitation and seizures. J Neurosci 34:2754-2763. CrossRef Medline
Zhang Z, Sun QQ (2011) The balance between excitation and inhibition and functional sensory processing in the somatosensory cortex. Int Rev Neurobiol 97:305-333. CrossRef Medline

Zoghbi HY, Bear MF (2012) Synaptic dysfunction in neurodevelopmental disorders associated with autism and intellectual disabilities. Cold Spring Harb Perspect Biol 4:a009886. CrossRef 